\title{
Non-equilibrium dissociating nitrogen flow over spheres and circular cylinders
}

\author{
By H. G. HORNUNG \\ Department of Physics, Australian National University, Canberra
}

(Received 23 October 1971)

Theoretical results based on the methods of Freeman and Garr \& Marrone show that the stand-off distance and flow pattern of non-equilibrium dissociating flow of nitrogen over the front part of a blunt body can be correlated in terms of a single reaction rate parameter $\Omega$ taking account of parameters describing the speed, density, dissociation and temperature of the free stream. The density pattern, which is sensitive to the reaction rate, consists of two distinct regions dominated by the effects of reaction and pressure respectively. The shape and size of these regions depend on $\Omega$. Experimental results obtained by optical interferometry in a free-piston shock tunnel confirm the theoretical results. A scale effect consistent with the induction time phenomenon suggested by Shui, Appleton \& Keck modifies the theoretical results considerably in the case of small models.

\section{Introduction}

The inviscid flow of nitrogen over a two-dimensional blunt body is considered in the case when the free-stream kinetic energy is comparable with the dissociation energy. If the internal energy of the free stream is small in comparison, the Mach number is high and the bow shock wave is strong. The shock converts the kinetic energy of the stream to heat, raising the temperature to a value where thermodynamic equilibrium requires the gas to dissociate. The dissociation rate is finite, however, so that there is a region behind the shock wave where the flow is not in equilibrium. The ambient conditions are taken to be such that the length scale introduced by the dissociation rate is comparable with the body radius.

The dissociation rate falls rapidly with temperature and therefore with shock slope. Thus the region affected by the chemical relaxation is limited to the vicinity of the stagnation point, the rate outside a 'cut-off' streamline being so slow that the composition remains constant for many body diameters downstream from the shock. If the body slope becomes small in a downstream direction, the temperature also decreases rapidly along streamlines originating in the stagnation region, which again causes the composition to freeze. Thus the region in which chemical relaxation occurs is restricted to the front part of the body.

In the limit of equilibrium flow, the dissociation rate is infinite and the relaxation zone becomes a thin sheet within the shock wave. As the reaction rate 
decreases, the relaxation region grows into a layer parallel to the shock which must eventually fill the whole shock layer, extending right up to the frozen boundaries.

The blunt body problem is mathematically complicated because the flow field contains a subsonic region, in which the equations of motion are elliptic, embedded in a supersonic field, where the equations are hyperbolic. Part of the boundary between these two regions is a sonic line of saddle-point singularities and the other part is the bow shock, along which some of the boundary conditions are given but the position of which, relative to the body, is not known a priori. The problem thus has to be solved numerically.

A number of methods exist for doing this. The one most commonly used is the inverse method, in which the shock shape is assumed known and the integration proceeds towards the body, whose shape is part of the solution. However, a simpler method was first applied to the problem with finite dissociation rate by Freeman (1958), who assumed the pressure field to be independent of the reaction rate and given by the Newton-Busemann result. He also assumed that the enthalpy and velocity were constant along streamlines, so that the problem was reduced to the solution of a set of ordinary differential equations. The inverse method was first applied to the non-equilibrium problem with fewer restrictive assumptions by Lick (1960) and developed to still more general situations by Garr \& Marrone (1963). The method of integral relations, in which conserved quantities are approximated by polynomials in one direction across the region, thus reducing the problem to a set of ordinary differential equations along the other direction, has also been applied to the non-equilibrium problem by Belotserkovskii \& Dushin (1964). More recently Kyriss (1970) adapted the time-dependent technique to non-equilibrium blunt body flows. This method starts from a known body shape and an approximation for the shock shape, and removes the difficulty of the unknown free boundary by introducing time as a further independent variable and letting conditions approach a steady state.

Although the later methods make few restrictive assumptions, and perhaps even because of this, they have not been used sufficiently widely to map out the dependence of the flow field on the various controlling parameters. Freeman's results present the general features of the flow in more detail than the later methods, in which the main aim was to develop a method of solution. There is thus a need to use one of these more refined techniques to study the dependence of the solution on a number of parameters. The aim of the first part of the present paper is to map out the dependence of numerical solutions to the non-equilibrium dissociating flow of nitrogen over spheres and circular cylinders on the relevant parameters, with a view to a correlation, and to delineate regions in which nonequilibrium dissociation affects the flow field significantly.

The importance of experimental results in an attempt to improve the understanding of non-equilibrium flow need not be emphasized. However, very few such results exist. Spurk \& Bartos (1966) presented interferograms of reacting flow of oxygen over cones, obtained in an expansion tube. Similar results were presented by Spurk (1970), giving a comparison with a theoretical model based on vibration-dissociation coupling. The difficulty in producing reacting flow in 
nitrogen, for which the dissociation energy is much larger than for oxygen, is that not only is it necessary to produce a stream of gas at a speed of about $5-8 \mathrm{~km} / \mathrm{s}$, but this stream has to be sufficiently dense and large for significant effects due to ohemical reaction to occur within one model diameter. This has only been achieved in a free-piston-driven shock tunnel so far, and an isolated case giving an interferogram of reacting flow over a cylinder was presented in Hornung (1971). It is clear that a much more complete experimental picture is needed if it is to be used for confirming theoretical models which involve many parameters. Even for a qualitative understanding of the variation of the flow field with reaction rate one would require a set of data covering a range of at least some of the relevant parameters.

The second aim of the present work is to conduct a systematic experimental investigation of reacting flow of nitrogen over a circular cylinder using a freepiston-driven shock tunnel and optical interferometry, and covering the maximum available range of the relevant parameters. The effect of free-stream nonequilibrium, which is a feature of devices in which the stream is accelerated by a nozzle expansion, and the influence of reaction rate on the flow are of particular interest.

\section{Theory}

\subsection{Equations of motion}

The conservation equations for steady inviscid compressible flow may be written as

$$
\begin{gathered}
\nabla \cdot(\rho \mathbf{q})=0, \\
(\mathbf{q} \cdot \nabla) \mathbf{q}+(\mathbf{1} / \rho) \nabla p=0, \\
(\mathbf{q} \cdot \nabla)\left(h+\frac{1}{2} q^{2}\right)=0,
\end{gathered}
$$

where $\mathbf{q}, \rho, p$ and $h$ are the velocity, density, pressure and specific enthalpy of the gas. $h$ is connected to the dynamic variables by an equation of state

$$
h=h(p, \rho, \alpha),
$$

where $\alpha$ is the mass fraction of atomic nitrogen. The problem is closed by the reaction rate equation, which may be written for the reaction

as

$$
\begin{gathered}
\mathrm{N}_{2}+\mathrm{M} \rightleftharpoons 2 \mathrm{~N}+\mathrm{M}, \quad \mathrm{M}=\mathrm{N} \text { or } \mathrm{N}_{2}, \\
\frac{D \alpha}{D t}=\left[2 k_{1} \alpha+(1-\alpha) k_{2}\right] \frac{\rho}{W}\left\{(1-\alpha)-\frac{4 \rho \alpha^{2}}{W K_{c}}\right\},
\end{gathered}
$$

where $W$ is the molecular weight of nitrogen $(=28), K_{c}$ is the equilibrium constant of the reaction (5) and $k_{1}$ and $k_{2}$ are the forward reaction rate constants for the reaction with $M=N$ and $M=N_{2}$ respectively. The equilibrium constant is defined by

$$
\alpha^{* 2} /\left(1-\alpha^{*}\right)=W K_{c} / 4 \rho,
$$

where $\alpha^{*}$ is the equilibrium dissociation fraction. The equilibrium and reaction rate constants are usually given as functions of the translational temperature $T$, which can be obtained from

$$
p / \rho=(R T / W)(1+\alpha),
$$

where $R$ is the universal gas constant. 
The boundary conditions on the shock wave are determined from the conservation of energy, momentum, mass and dissociation fraction across the shock:

$$
\left.\begin{array}{c}
h_{\infty}+\frac{1}{2} u_{\infty}^{2}=h_{s}+\frac{1}{2} q_{s}^{2}, \quad p_{\infty}+\rho_{\infty} u_{n \infty}^{2}=p_{s}+\rho_{s} q_{n s}^{2}, \\
\rho_{\infty} u_{n \infty}=\rho_{s} q_{n s}, \quad \alpha_{\infty}=\alpha_{s},
\end{array}\right\}
$$

the subscript $n$ referring to components normal to the shock. These have to be solved iteratively for the pressure, density and velocity on the downstream side of the shock unless a simple form is assumed for the equation of state (4). The boundary condition on the body requires the velocity to be tangential to the surface.

In the present work this problem is solved by Freeman's method as well as by Garr \& Marrone's method. The advantages of Freeman's method are first, that its relative simplicity makes it possible to see the way in which certain features of the solution come about, and second, that the computation time required is relatively small. The latter is an important consideration here because a large number of computations have to be made. It is necessary, however, to check that the results obtained with this approximate technique describe the features of the flow field correctly. For this purpose Garr \& Marrone's inverse method is used. While there are many arguments against the use of inverse methods, they have the advantage over the method of integral relations that the solution is given in detail throughout the flow field, whereas in the method of integral relations a considerable increase in computing time is necessary to obtain anything more than data along two boundaries (usually shock and body). The time-dependent method combines the advantages of both techniques but became available too late for this work.

\subsection{Freeman's method}

The assumption which makes it possible to separate the pressure field from the remaining problem in Freeman's method is that the density in the shock layer is so much larger than the free-stream density that quantities of the order of the inverse density ratio can be neglected compared with unity. This leads to a thin shock layer in which the streamlines are parallel to the body and in which the momentum equations (2) reduce to

$$
q=q(\psi), \quad \partial p / \partial \psi=q \kappa,
$$

where $q$ is the flow speed, $\kappa$ is the streamline curvature and $\psi$ is the stream function. The pressure can thus be obtained directly by integrating (10) to give an expression of the form

$$
p=p(x, \psi)
$$

where $x$ is the streamwise co-ordinate and where the shock boundary condition for pressure (9) is used in the integration. The energy equation (3) simplifies to

$$
h=h(\psi) \text {. }
$$

The enthalpy is taken to be of the form

$$
W h / R \theta_{d}=(4+\alpha) T / \theta_{d}+\alpha
$$


which gives the enthalpy for a Lighthill (1957) ideal dissociating gas, $\theta_{d}$ being the characteristic temperature of dissociation. The boundary condition for $h$ at the shock is then

$$
\frac{W h_{s}}{R \theta_{d}}=\left(4+\alpha_{\infty}\right) \frac{T_{\infty}}{\theta_{d}}+\frac{W u_{\infty}^{2}}{2 R \theta_{d}} \sin ^{2} \beta+\alpha_{\infty},
$$

where $\beta$ is the angle between the free stream and the shock, and the component of velocity normal to the shock has been neglected in the shock layer. Thus

$$
q_{s}=u_{\infty} \cos \beta \text {. }
$$

The temperature may now be obtained as an explicit function of $\alpha$ and freestream conditions, for a given streamline defined by the value of $\beta$ at which the streamline crossed the shock, by using (12), (13) and (14):

$$
\frac{T}{\theta_{d}}=\frac{1}{4+\alpha}\left[\left(4+\alpha_{\infty}\right) \frac{T_{\infty}}{\theta_{d}}+\mu \sin ^{2} \beta-\left(\alpha-\alpha_{\infty}\right)\right],
$$

where $\mu$ is the dimensionless parameter $W u_{\infty}^{2} / 2 R \theta_{d}$. Equations (16), (11) and (8) then yield a similar expression for the density:

$$
\frac{\rho}{\rho_{\infty}}=\frac{2 p \mu}{\rho_{\infty} u_{\infty}^{2}(1+\alpha)} \frac{\theta_{d}}{T}
$$

The square bracket in (6) is written as

$$
2 k_{1} \alpha+(1-\alpha) k_{2}=W C T^{\eta} e^{-\theta_{d} / T},
$$

thus incorporating the two components of reaction rate into one constant $C$. This, however, is not a serious restriction in view of the uncertainty associated with experimental data on reaction rates for nitrogen. Writing the equilibrium constant in Lighthill's form,

$$
\frac{\alpha^{* 2}}{1-\alpha^{*}}=\frac{\rho_{d}}{\rho} e^{-\theta_{d} / T},
$$

with $\rho_{d}$ constant, the reaction rate equation is

$$
\frac{d \alpha}{d s}=\frac{C \rho T^{\eta}}{q}\left\{(1-\alpha) e^{-\theta_{d l} / T}-\frac{\rho}{\rho_{d}} \alpha^{2}\right\}
$$

for a particular streamline. Here $d s$ is the length element along a streamline, which for the thin shock layer on a body of circular section is $a d \phi$, where $a$ is the body radius and $\phi$ is the angle measured from the stagnation streamline. Using (16) and (17), the right-hand side of (20) may be written as a function of $\alpha$, so that a direct integration gives $\alpha$ as a function of $\phi$ for a particular streamline. Equation (17) then gives the density, and the normal distance of the streamline from the body can be evaluated from

$$
y=\int_{0}^{\psi} \frac{d \psi}{\rho q(a \sin \phi)^{j}}
$$

where $j=0$ or 1 for plane or axisymmetric flow respectively. The integral in (21) does not converge for small values of $\psi$ in the plane case. This can be 
overcome by applying the following approximate correction to equation (15) (see Freeman 1958):

$$
q=u_{\infty}\left(\cos ^{2} \beta-\frac{2 \rho_{\infty}}{\rho} \log \frac{p_{b}}{\rho_{\infty} u_{\infty}^{2}}\right)^{\frac{1}{2}},
$$

where $p_{b}$ is the pressure on the body as given by (11) with $q$ in the form of (15). Applying this correction gives results for the plane case which are qualitatively similar to those given in the following section for the axisymmetric case. This was also the method used in Hornung (1971) for comparison with experiment. The results from calculations for the plane case using the Freeman method are not presented here, however, since this would make the present paper unnecessarily large.

The parameters controlling the above problem are those describing the free stream, namely $\mu, \alpha_{\infty}, \rho_{d} / \rho_{\infty}, T_{\infty} / \theta_{d}$, and those describing the reaction rate, namely $\eta$ and $2 a C \rho_{\infty} \theta_{d}^{\eta} / u_{\infty}=\Lambda$. The solutions given by Freeman (1958) are for flow over a sphere in the case $\alpha_{\infty}=T_{\infty}=\eta=0$, with main emphasis on the effect of $\Lambda$. He observed that the profiles of $\alpha$ across the shock layer are almost independent of $\phi$ although they vary considerably with $\Lambda$. The following section presents results of numerical solutions investigating the dependence on all the above parameters, but restricting $T_{\infty} / \theta_{d}$ to small values in order to keep the free-stream Mach number large.

\subsection{Numerical results obtained by Freeman's method}

One of the parameters of interest in the blunt body problem is the shock wave stand-off distance. In the frozen or equilibrium limit this depends mainly on the density ratio across the shock, being inversely proportional to it. Thus one might expect the free-stream dissociation to have a strong influence on the stand-off distance $\Delta$, since the density ratio in the frozen limit varies approximately from 7 to 4 , corresponding to a change of $\alpha_{\infty}$ from 0 to 1 . A selection of values of $\Delta / a$ resulting from numerical calculations of the axisymmetric case is presented in figure 1 as a plot against $\log \Lambda$ for three values of $\alpha_{\infty}$ and $\eta$. Freeman's results for $\eta=\alpha_{\infty}=T_{\infty}=0, \mu=1$ are superimposed in figure 1 as a check. It is immediately clear that the variation of $\Delta / a$ with $\alpha_{\infty}$ is sufficient for a change from $\alpha_{\infty}=0$ to $0 \cdot 4$ to offset the variation due to a change in $\Lambda$ from 0 to $\infty$.

There appears to be a systematic dependence of these results on the parameter $\eta$, in that curves for larger $|\eta|$ are displaced to the left by a shift proportional to

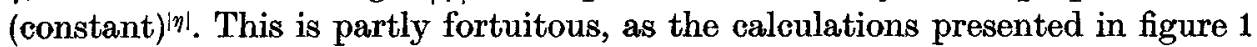
are made at conditions where the temperature immediately downstream of the shock is almost the same for all calculations. Thus the dissociation rate immediately after the shock, which is proportional to $\Lambda\left(T / \theta_{d}\right)^{\eta} e^{-\theta_{d} / T_{s}}\left(1-\alpha_{\infty}\right)$, could be written as

$$
\left[\Lambda \exp \left(\eta \log T_{s} / \theta_{d}\right)\right] \exp \left(-\theta_{d} / T_{s}\right)\left(1-\alpha_{\infty}\right)
$$

showing the dependence of an effective reaction rate parameter, in square brackets, on $\eta$. While this is not useful in itself, it suggests that the dissociation rate immediately after the normal shook may be a sufficiently precise quantity to characterize at least overall results such as the stand-off distance. 


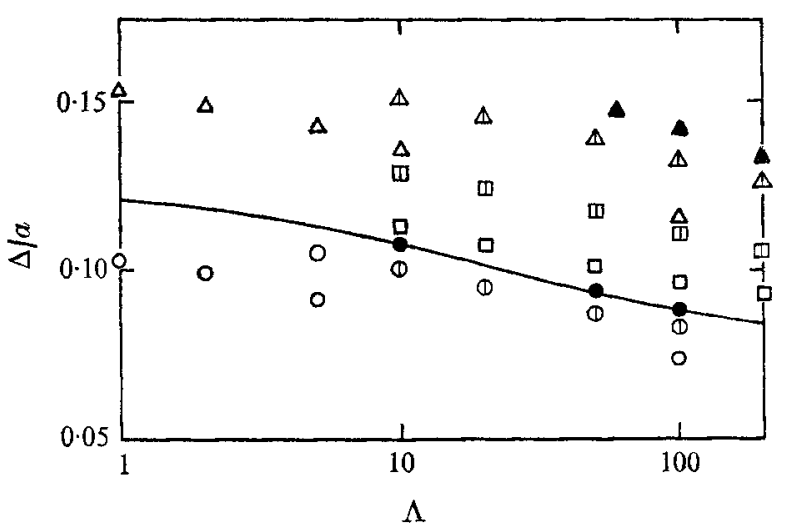

FIGURE 1. Shock stand-off distance on spheres as calculated by Freeman method. $0, \alpha_{\infty}=0$; $\square, \alpha_{\infty}=0.2 ; \Delta, \alpha_{\infty}=0.4$; filled symbol, $\eta=0$; crossed symbol, $\eta=-0.5$; open symbol, $\eta=-1.5 ;-$ Freeman's curve, $\alpha_{\infty}=\eta=T_{\infty} / \theta_{a}=0$.

In Freeman's approximation the temperature and density immediately behind the normal shock may be expressed directly in terms of the free-stream conditions from (16) and (17) with $\beta=\frac{1}{2} \pi, \alpha=\alpha_{\infty}$ and $p / \rho_{\infty} u_{\infty}^{2}=1$ as

$$
\frac{T_{s}}{\theta_{d}}=\frac{T_{\infty}}{\theta_{d}}+\frac{\mu}{4+\alpha_{\infty}} \quad \text { and } \quad \frac{\rho_{s}}{\rho_{\infty}}=\frac{2 \mu}{\left(1+\alpha_{\infty}\right)} \frac{\theta_{d}}{T_{s}}
$$

Thus the dissociation rate (20) can be written in terms of free-stream conditions directly:

$$
\left(\frac{d \alpha}{d t}\right)_{s}=2 \mu \Lambda \frac{\left(1-\alpha_{\infty}\right)}{\left(1+\alpha_{\infty}\right)}\left[\frac{T_{\infty}}{\theta_{d}}+\frac{\mu}{4+\alpha_{\infty}}\right]^{\eta-1} \exp \left[\left(-\frac{T_{\infty}}{\theta_{d}}-\frac{\mu}{4+\alpha_{\infty}}\right)^{-1}\right],
$$

where the recombination rate has been neglected. The ratio of this rate to the flow rate $u_{\infty} / a$ is now denoted as

$$
\Omega=\left(\frac{d \alpha}{d t_{s}}\right) \times \frac{a}{u_{\infty}}
$$

the normal shock reaction rate parameter.

The results of seventy calculations of the axisymmetric case are presented in figure 2 as a plot of $(\Delta / 2 a)\left(\rho_{s} / \rho_{\infty}\right) v s . \log \Omega$, the ranges of values of the parameters $\Lambda, \eta,\left(\rho_{\infty} / \rho_{d}\right) \mu, \alpha_{\infty}$ and $T_{\infty} / \theta_{d}$ being given in table 1 . It is seen that this dimensionless grouping of the free-stream and reaction rate parameters, that is $\Omega$, correlates the stand-off density product quite well. It should be pointed out that the improvement in the correlation of the data compared with figure 1 is partly accomplished by including the density ratio in the stand-off parameter, but, even for the limited range of conditions presented in figure 1, a correlation based on $\Lambda$ instead of $\Omega$ would be quite unsatisfactory.

The detailed flow field can best be presented in the form of contour plots of $T, p, \alpha$ and $\rho$. This is done for a particular case in figure 3 , where all these contours are superimposed in a plot of the normal distance from the body versus the angle from the stagnation point, $\phi$. The contours of pressure, which run predominantly 


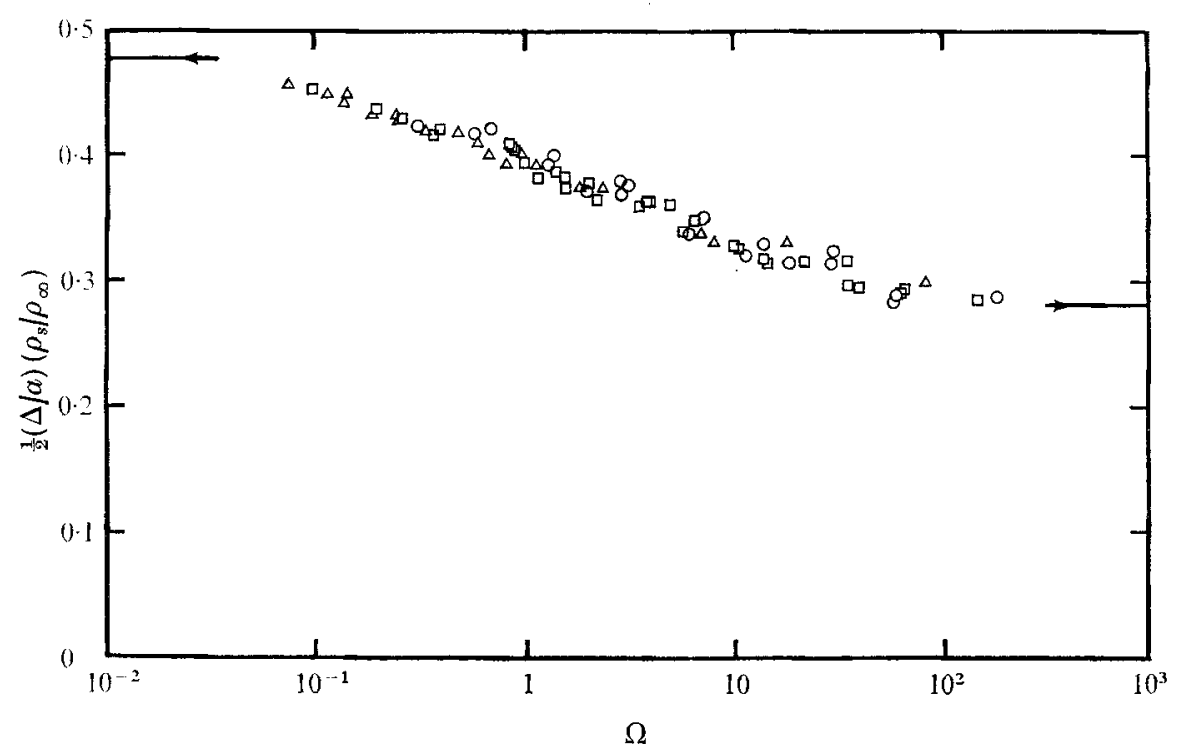

FIGURE 2. Correlation of stand-off distance with reaction rate parameter. Calculations by Freeman method for spheres. $O, \alpha_{\infty}=0 ; \square, \alpha_{\infty}=0.2 ; \Delta, \alpha_{\infty}=0.4 ; \rightarrow$, asymptote.

\begin{tabular}{|c|c|c|c|c|c|c|}
\hline Parameter & $\Lambda$ & $\eta$ & $\alpha_{\infty}$ & $\mu$ & $T_{\infty} / \theta_{\boldsymbol{d}}$ & $\rho_{d} \mid \rho_{\infty}$ \\
\hline Range $\left\{\begin{array}{l}\text { from } \\
\text { to }\end{array}\right.$ & $\begin{array}{r}1 \\
1000\end{array}$ & $\begin{array}{c}0 \\
-1.5\end{array}$ & $\begin{array}{l}0 \\
0 \cdot 4\end{array}$ & $\begin{array}{l}0 \cdot 5 \\
1 \cdot 0\end{array}$ & $\begin{array}{l}0 \\
0.02\end{array}$ & $\begin{array}{l}10^{6} \\
10^{8}\end{array}$ \\
\hline
\end{tabular}

TABLE 1. Range of values of parameters covered in calculation by Freeman's method (sphere)

at right angles to the shock layer, are determined by equations (11) and (21) and are only weakly dependent on the reaction rate through the density term in (21). They can thus be expected to be similar for other conditions. The contours of $\alpha$ and $T$ are seen to run predominantly parallel to the shock layer. This may be expected in a region where the dissociation rate is very large, for the following reasons. The primary cause of temperature change in such a region is the dissociation which uses up energy and therefore lowers the temperature. Thus the temperature gradient is approximately parallel but opposite in direction to $\nabla \alpha$. However, in the Freeman approximation $h$ is constant along streamlines and since $h$ is a function only of $\alpha$ and $T, \nabla h$ (and therefore $\nabla \psi$ ) must be approximately parallel to $\nabla \alpha$ and $\nabla T$. Now in the major part of the thin shock layer the streamlines run parallel to the body. Thus the contours of $\alpha$ and $T$ can be expected to be approximately parallel to the body in a region of strong dissociation. Where the streamlines do not run parallel to the body, such as in the immediate vicinity of the stagnation point, the enthalpy gradient is so small that the effects on $\nabla h$ of $\nabla \alpha$ and $\nabla T$ must balance each other anyway.

The most interesting variable to study, however, is the density because it is easily related to the fringe shift in an optical interferogram and because the 


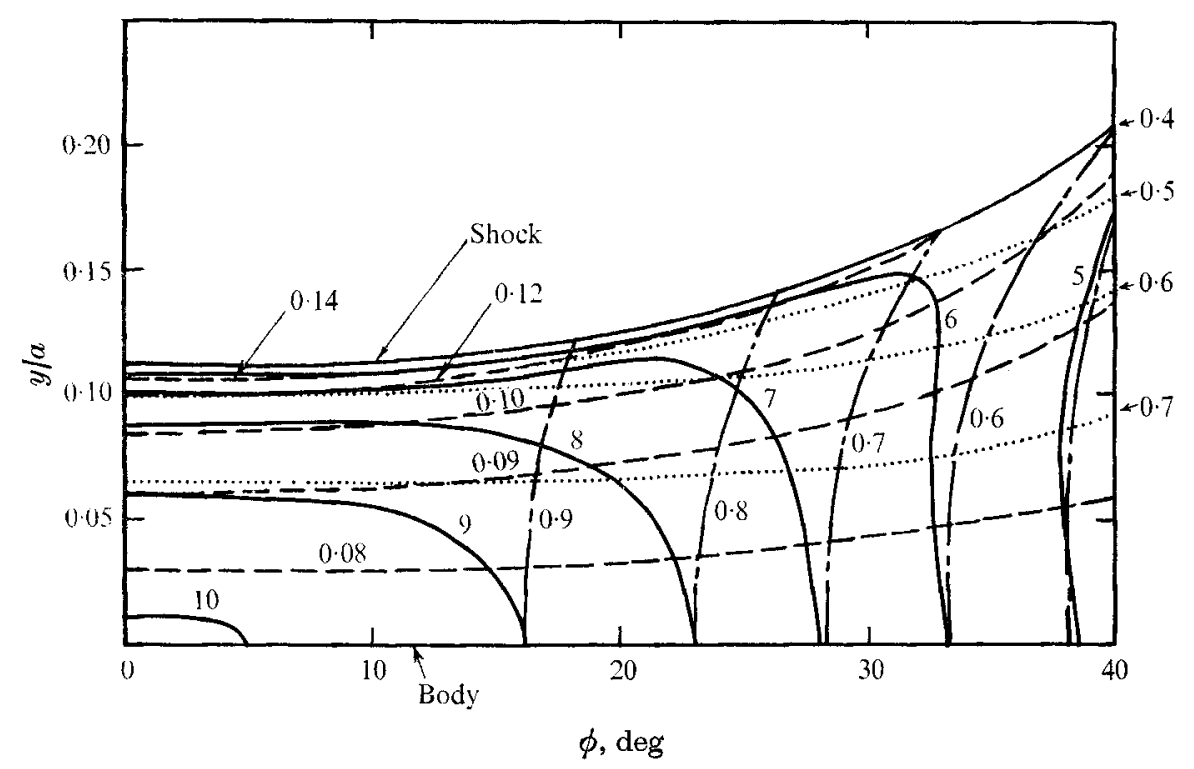

Figure 3. Temperature, pressure, dissociation and density fields in the shock layer of a sphere as calculated by Freeman method. _-, $\left.\rho / \rho_{\infty} ;-.-, p / \rho_{\infty} u_{\infty}^{2} ; \cdots, \alpha ; \ldots, T\right] \theta_{d}$. $\mu=0.7, \alpha_{\infty}=0.4, \rho_{d} / \rho_{\infty}=10^{7}, T_{\infty} / \theta_{a}=0.01, \eta=-1.5, \Omega=7$.

density field is quite sensitive to the reaction rate. The latter feature is illustrated in figure 4, in which three plots like those in figure 3 are presented, corresponding to slow, medium and fast reaction rates. Again, the $\alpha$ and $T$ contours run parallel to the body except that for the fast reaction they are crowded near the shock and for the slow reaction they are crowded near the body. The pressure contours again run predominantly at right angles to the shock layer. However, the density contours are quite markedly different in the three situations. If the above broad features of the $\alpha, T$ and $p$ contours are kept in mind, it is relatively simple to see how this change in the density pattern comes about, by considering equation (8).

$\nabla \rho$ may be thought of as the resultant of $\nabla p$, which is parallel to the shock layer, and of the gradients of $\alpha$ and $T$, which are normal to the shock layer. The direction of $\nabla \rho$ is thus parallel to $\nabla \alpha$ and $\nabla T$, that is, parallel to the body, if $\nabla \alpha$ and $\nabla T$ are large and $\nabla p$ is small. Similarly $\nabla \rho$ is parallel to $\nabla p$ if $\nabla \alpha$ is much smaller than $\nabla p$. The cause of the spectacular change in the density pattern with increasing reaction rate is thus due mainly to the shift of the high $\alpha$ gradient from the region near the body to a layer near the shock. Wherever the dissociation fraction is nearly constant the density and pressure patterns are thus very similar. That is, in the frozen-flow limit the density pattern will be like the pressure pattern; as the reaction rate is increased, the density pattern will depart from the form of the pressure pattern to become similar to that of figure $4(b)$ and on further increase in the reaction rate the form of the pattern reverts back to that of the pressure pattern in the near-equilibrium limit, but the general level of the density is then much higher. The fact that the two important effects on density are acting approximately at right angles to each other thus makes it possible to study their influence separately. This is therefore a case where a 
$\phi, \operatorname{deg}$
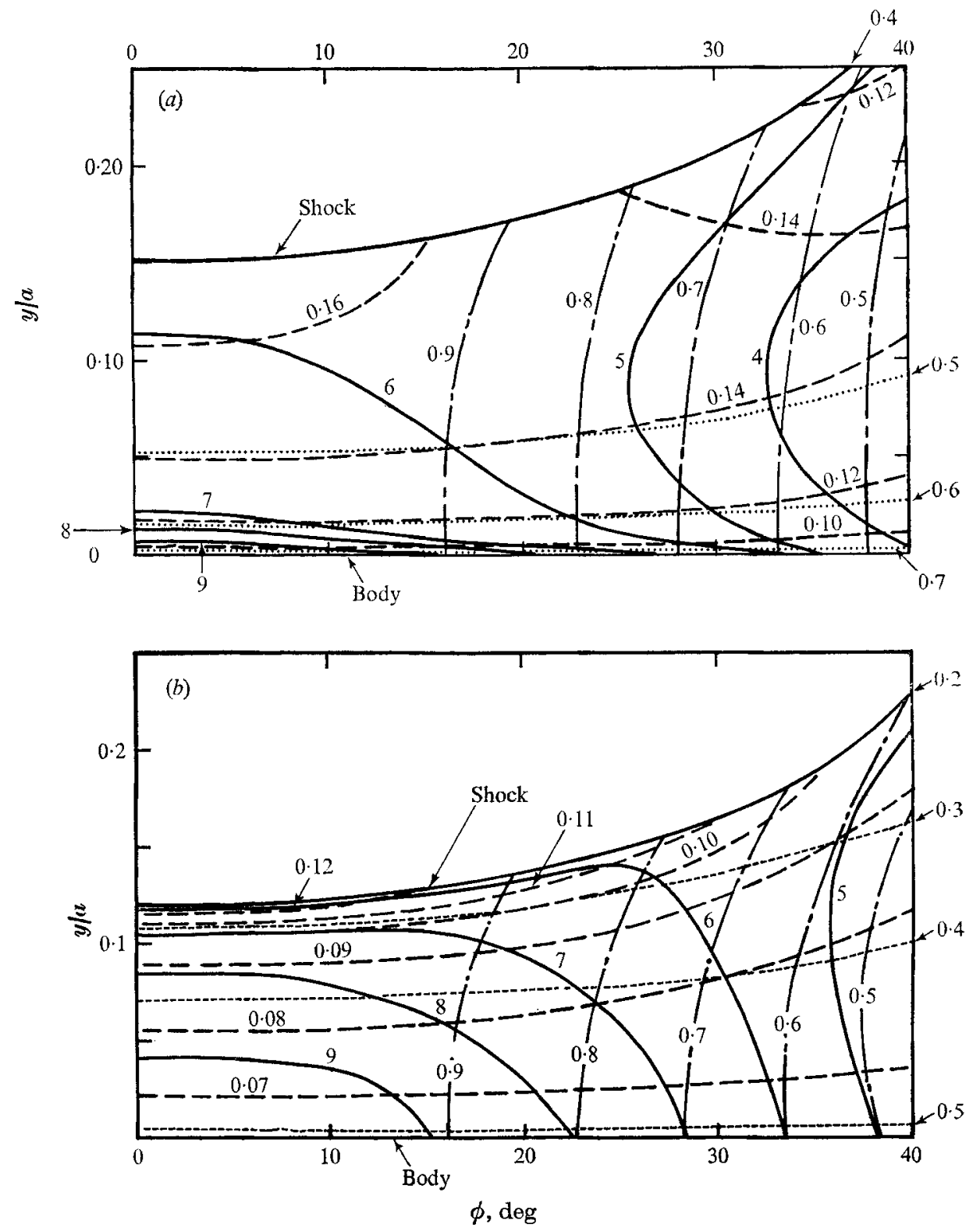

FIGURe 4(a), (b). For legend see facing page.

two-dimensional flow field helps to understand the problem more effectively than a one-dimensional one.

Figure 5 shows a perspective view of three density fields with density plotted vertically over the $y / \Delta, \phi$ plane. The upper and lower sheets represent the cases of equilibrium and frozen flow respectively and are very similar in shape. The intermediate sheet represents the case with finite reaction rate, with very strong variation in density and quite a different shape. 


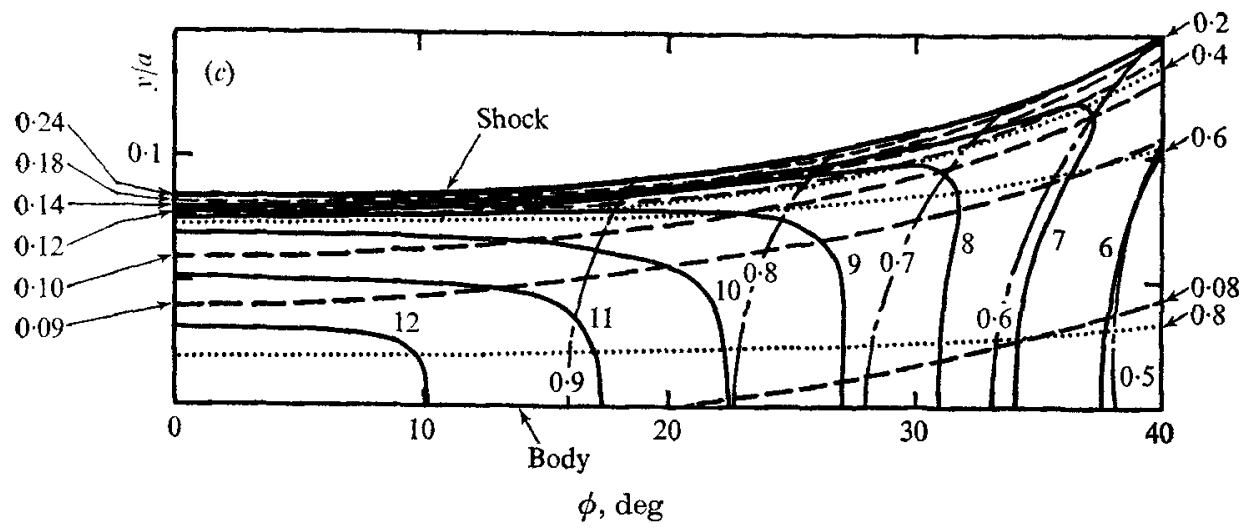

FIGURE 4. Flow field near the stagnation point of a sphere. Freeman method. - - $\rho / \rho_{\infty}$; $-\cdots, p / \rho_{\infty} u_{\infty}^{2} ; \cdots, \alpha ;--, T / \theta_{d}$. (a) Slow reaction, $\mu=0 \cdot 7, \alpha_{\infty}=0.4, \rho_{d} / \rho_{\infty}=10^{7}$, $T_{\infty} / \theta_{d}=0.01, \Lambda=10, \eta=-0.5, \Omega=0.12$. (b) Medium reaction, $\mu=0.5, \alpha_{\infty}=0.2$, $d / \rho_{\infty}=10^{7}, T_{\infty} / \theta_{d}=0 \cdot 02, \Lambda=100, \eta=-1 \cdot 5, \Omega=3 \cdot 5$. (c) Fast reaction, $\mu=1 \cdot 0, \alpha_{\infty}=0 \cdot 2$, $\rho_{d} \mid \rho_{\infty}=10^{7}, T_{\infty} / \theta_{d}={ }^{\prime} 0, \Lambda^{\prime}=100, \eta=-1 \cdot 5, \Omega=36$.

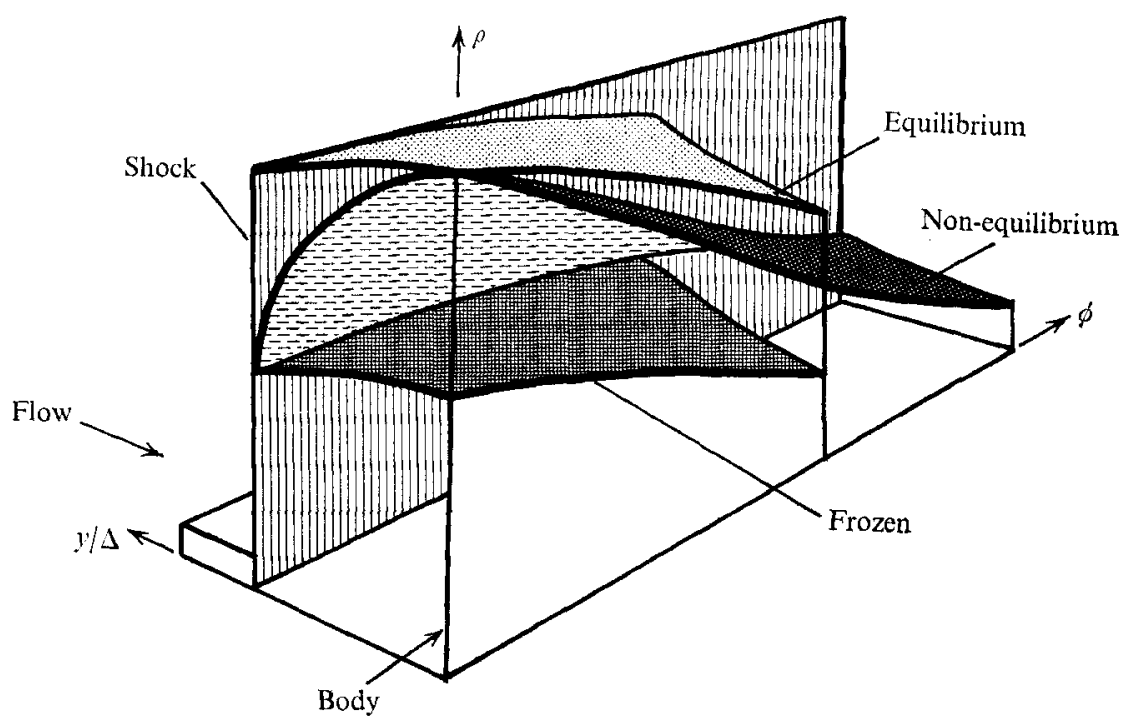

FIGURE 5. Three-dimensional sketch of density surface in the shock layer of a blunt body for equilibrium, frozen and non-equilibrium flow.

To test the effectiveness of $\Omega$ as a correlating parameter, three pairs of cases at low, medium and high values of $\Omega$ are selected for figure 6 , showing density patterns in $y, \phi$ co-ordinates as in figure 4 . The parameters $\Lambda, \mu, \eta, \alpha_{\infty}, T_{\infty} / \theta_{d}$ and $\rho_{\infty} / \rho_{d}$ are all different for the separate pairs but give approximately the same value of $\Omega$. It is seen that the density patterns are very similar if $\Omega$ is the same and differ considerably for different values of $\Omega$. Thus both the density pattern, which is very sensitive to the dissociation rate, and the product of stand-off distance and density ratio are correlated by the dissociation rate immediately behind the normal shock. 


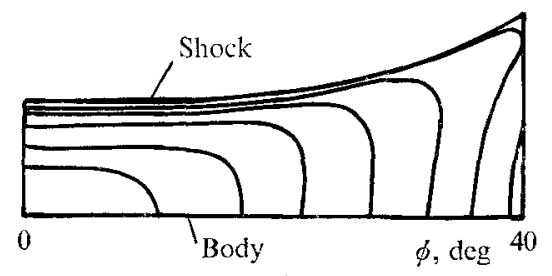

$(0)$

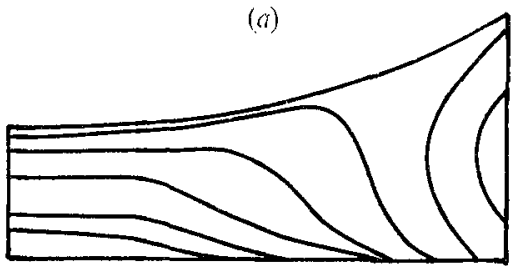

(c)

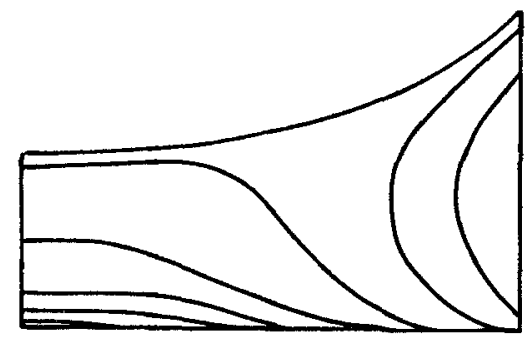

(e)

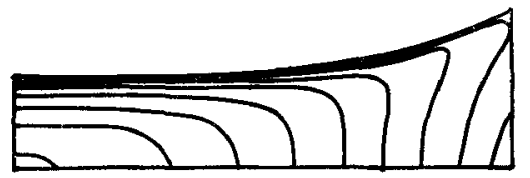

(b)

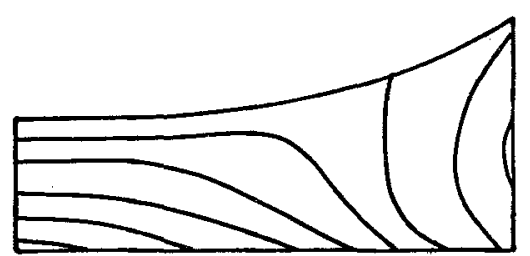

(d)

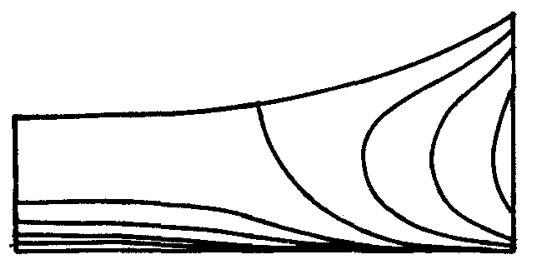

$(f)$

FIGURE 6. Three pairs of density fields with similar values of $\Omega$, but widely different sets of the six parameters.

\begin{tabular}{|c|c|c|c|c|c|c|c|}
\hline & $\Omega$ & $\mu$ & $\alpha_{\infty}$ & $\left.\rho_{d}\right] \rho_{\infty}$ & $T_{\infty} / \theta_{d}$ & $\Lambda$ & $-\eta$ \\
\hline$(a)$ & 41 & 1.0 & 0.2 & $10^{7}$ & 0.02 & 100 & $1 \cdot 5$ \\
\hline (b) & 58 & 1.0 & 0 & $10^{7}$ & 0 & 100 & $1 \cdot 5$ \\
\hline (c) & 3.9 & 0.7 & 0.2 & $10^{7}$ & 0.05 & 200 & 0.5 \\
\hline (d) & $4 \cdot 3$ & 1.0 & 0.2 & $10^{6}$ & 0 & 50 & 0.5 \\
\hline (e) & 0.59 & 0.7 & 0.4 & $10^{7}$ & $0 \cdot 01$ & 50 & 0.5 \\
\hline$(f)$ & 0.57 & 1.0 & 0 & $10^{7}$ & 0 & 1 & 1.5 \\
\hline
\end{tabular}

To test that these features of the solution, and particularly their correlation, are not the result of built-in conditions in the simplicity of Freeman's model, the following section presents results obtained with Garr \& Marrone's inverse method.

\subsection{Garr \& Marrone's method and results}

Garr \& Marrone (1963) considered the full equations of motion, (1)-(4) and (6) -(8), and their method is written for more general rate processes than the simple ones considered here (equation (6)) for the symmetrical diatomic gas. Their model includes provision for vibrational relaxation and for mixtures of reacting gases. Equations (4) and (7), specifying the thermodynamics of the gas, are determined from a detailed model at each point, providing both enthalpy and 'specific heat'. This calculation is set up to include the effects of equilibrium electronic excitation. In the present use of the method rotation, vibration and electronic excitation are assumed to be in thermodynamic equilibrium with the translational temperature and the effects of ionization are neglected. 
The method proceeds by solving equations (9) iteratively for a known shock shape, in this case always taken to be a catenary defined by its maximum curvature, and then integrating inwards along rays normal to the shock. Successive rays are terminated as they enter the body, that is, as the mass flow rate across the ray approaches that across the shock between the ray and the axis. A sliding seven-point fit to the values of the variables on the ends of the rays determines the approximate analytic form of the variables, thus giving the derivatives parallel to the shock, which are needed to determine the derivatives along a streamline. The method is written for the axisymmetric case, but is easily modified to the plane case by altering the symmetry of the continuity equation and changing the form of the mass flow condition.

With the more complicated gas model of this method, the number of parameters needed to define the equilibrium condition of the gas is, of course, much larger than for the Lighthill gas. As well as this the reaction is taken to be more complicated in this section, the square bracket of equation (6) being left general, with the reaction rate constants of the form

$$
k_{1}=C_{1} T^{\eta_{1}} e^{-\theta_{d} \mid T}, \quad k_{2}=C_{2} T^{\eta_{2}} e^{-\theta_{d} \mid T} .
$$

Even if the additional parameters involved in the equilibrium condition are disregarded, there are then two more parameters needed to describe the reaction rate and the separate examination of the influence of each of the parameters on the problem would require a prohibitive amount of computer time. Garr \& Marrone's method is therefore only applied to seventeen cases of flow over a sphere, using reaction rates as recommended by Cary (1965). These cases are listed in table 2 , in which are also given the parameter $\Omega$, from equations (6), (24) and (25), as well as the ratio $\Gamma$ of the contributions to the reaction rate from the reaction with $\mathrm{M}=\mathrm{N}$ to that with $\mathrm{M}=\mathrm{N}_{2}$ in equation (5):

$$
\Gamma=2 k_{1} \alpha /(1-\alpha) k_{2} .
$$

The product of stand-off distance and density ratio is again plotted against $\Omega$ for these results in figure 7 . It is seen that the correlation is confirmed, and no significant sensitivity to the parameter $\Gamma$, which is varied by a factor of 30 , is evident in the stand-off distances. Also plotted in figure 7 are the asymptotic values of the ordinate for $\Omega=0$ and $\infty$. These are calculated by Lomax \& Inouye's (1964) inverse method, which uses a slightly different grid, refers to gas tables for the equilibrium condition and iterates on the shock shape until the body shape is within a specified error of the desired shape. It is seen that these asymptotes fit the trend of the reacting flow cases quite well. It should be pointed out here that the body shape obtained with a catenary shock by Garr \& Marrone's method is not exactly spherical but the radius of curvature is always constant to within $\pm 3 \%$ over the calculated portion of the body for spheres and $\pm 5 \%$ for cylinders.

By comparing figure 7 with figure 2 , it can be seen that while the two sets of results agree qualitatively the curve given by Freeman's method is higher by about $30 \%$. This is true also for the asymptotic values of the two figures, and is thought to be due to the fact that the velocity distribution across the shock layer 


\begin{tabular}{|c|c|c|c|c|c|c|}
\hline$\alpha_{\infty}$ & $\underset{\mathrm{km} \mathrm{s}^{-1}}{V_{\infty}}$ & $\begin{array}{c}\rho_{\infty} \times 10^{6} \\
\mathrm{~g} \mathrm{~cm}^{-3}\end{array}$ & $T_{\infty}{ }^{\circ} \mathbf{K}$ & $a \mathrm{~cm}$ & $\Omega$ & $\Gamma$ \\
\hline 0.073 & $5 \cdot 683$ & $2 \cdot 424$ & 1442 & 0.0155 & 0.0132 & $0 \cdot 172$ \\
\hline 0.073 & $5 \cdot 683$ & $2 \cdot 442$ & 1442 & 0.0627 & 0.054 & $0 \cdot 172$ \\
\hline $0 \cdot 073$ & $5 \cdot 683$ & $2 \cdot 424$ & 1442 & $0 \cdot 250$ & $0 \cdot 123$ & $0 \cdot 172$ \\
\hline 0.073 & $5 \cdot 683$ & $2 \cdot 424$ & 1442 & $1 \cdot 03$ & 0.88 & 0.172 \\
\hline 0.073 & $5 \cdot 683$ & $2 \cdot 424$ & 1442 & $4 \cdot 15$ & $3 \cdot 56$ & $0 \cdot 172$ \\
\hline 0.073 & $5 \cdot 683$ & $2 \cdot 424$ & 1442 & $12 \cdot 2$ & $14 \cdot 3$ & $0 \cdot 172$ \\
\hline 0.073 & $5 \cdot 683$ & $2 \cdot 424$ & 1442 & $48 \cdot 8$ & $57 \cdot 4$ & 0.172 \\
\hline 0.424 & 6.998 & $1 \cdot 576$ & 1818 & 0.015 & 0.031 & $0 \cdot 488$ \\
\hline $0 \cdot 424$ & 6.998 & $1 \cdot 576$ & 1818 & 0.063 & $0 \cdot 127$ & 0.488 \\
\hline 0.424 & $6 \cdot 998$ & $1 \cdot 576$ & 1818 & $0 \cdot 255$ & 0.514 & 0.488 \\
\hline $0 \cdot 424$ & $6 \cdot 998$ & $1 \cdot 576$ & 1818 & $1 \cdot 04$ & $2 \cdot 06$ & 0.488 \\
\hline $0 \cdot 424$ & $6 \cdot 998$ & $1 \cdot 576$ & 1818 & $4 \cdot 51$ & $9 \cdot 1$ & 0.488 \\
\hline 0.424 & $6 \cdot 998$ & 1.576 & 1818 & $18 \cdot 3$ & 37 & $0 \cdot 488$ \\
\hline 0.424 & $6 \cdot 998$ & 1.576 & 1818 & 74 & 150 & 0.488 \\
\hline $0 \cdot 656$ & $8 \cdot 94$ & 0.878 & 1617 & 0.255 & 0.64 & $5 \cdot 46$ \\
\hline 0.656 & 8.94 & 0.878 & 1617 & $1 \cdot 06$ & $2 \cdot 67$ & $5 \cdot 46$ \\
\hline $0 \cdot 656$ & $8 \cdot 94$ & 0.878 & 1617 & $4 \cdot 18$ & $10 \cdot 5$ & $5 \cdot 46$ \\
\hline
\end{tabular}

TABLE 2. Conditions for calculations made by Garr \& Marrone's method (spheres). For all these calculations the following reaction rate constants were used:

$C_{1}=7.1 \times 10^{19} \mathrm{~cm}^{3} \mathrm{~mol}^{-1} \mathrm{~s}^{-1}, C_{2}=5.6 \times 10^{22} \mathrm{~cm}^{3} \mathrm{~mole}^{-1} \mathrm{~s}^{-1}, \eta_{1}=1 \cdot 0, \eta_{2}=-1 \cdot 7$.

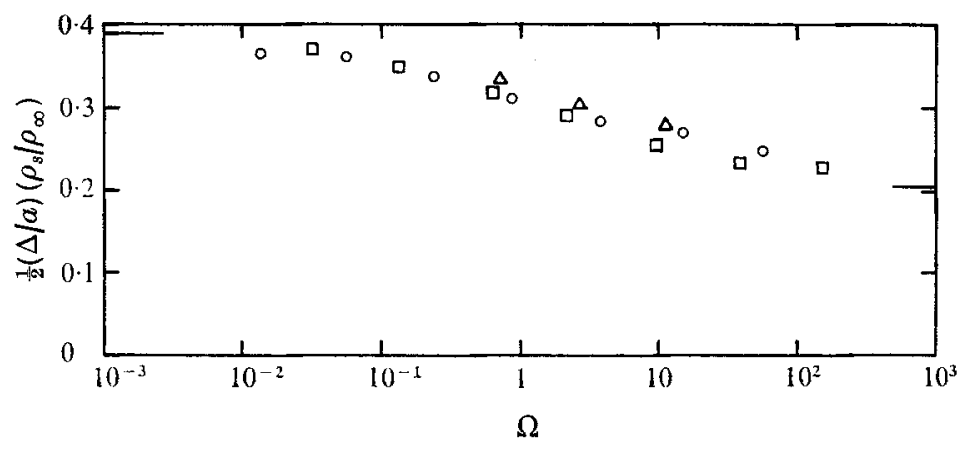

Fraure 7. Correlation of stand-off distance on spheres with reaction rate parameter. Calculations by Garr \& Marrone's method. $O, \alpha_{\infty}=0.07 ; \square, \alpha_{\infty}=0.42 ; \Delta, \alpha_{\infty}=0.65$; —, asymptotes calculated by Lomax \& Inouye's method.

in the Freeman model is lower than in the Garr \& Marrone or the Lomax \& Inouye model, being zero on the body, where the density is highest. Thus the displacement thickness of the inner shock layer can be expected to be overestimated by the Freeman method.

The density patterns of seven selected cases, including the frozen and equilibrium cases, are plotted next to each other in ascending order of $\Omega$ in figure 8 . It is seen that the pattern alters qualitatively in the same way as in figure 4.

In all the features described above, the flow over circular cylinders is very similar to that over spheres. This is illustrated by figure 9 , in which are shown the density fields of seven cases in ascending order of $\Omega$, the values of which are separated by a factor of four except for the first and last cases, which represent 


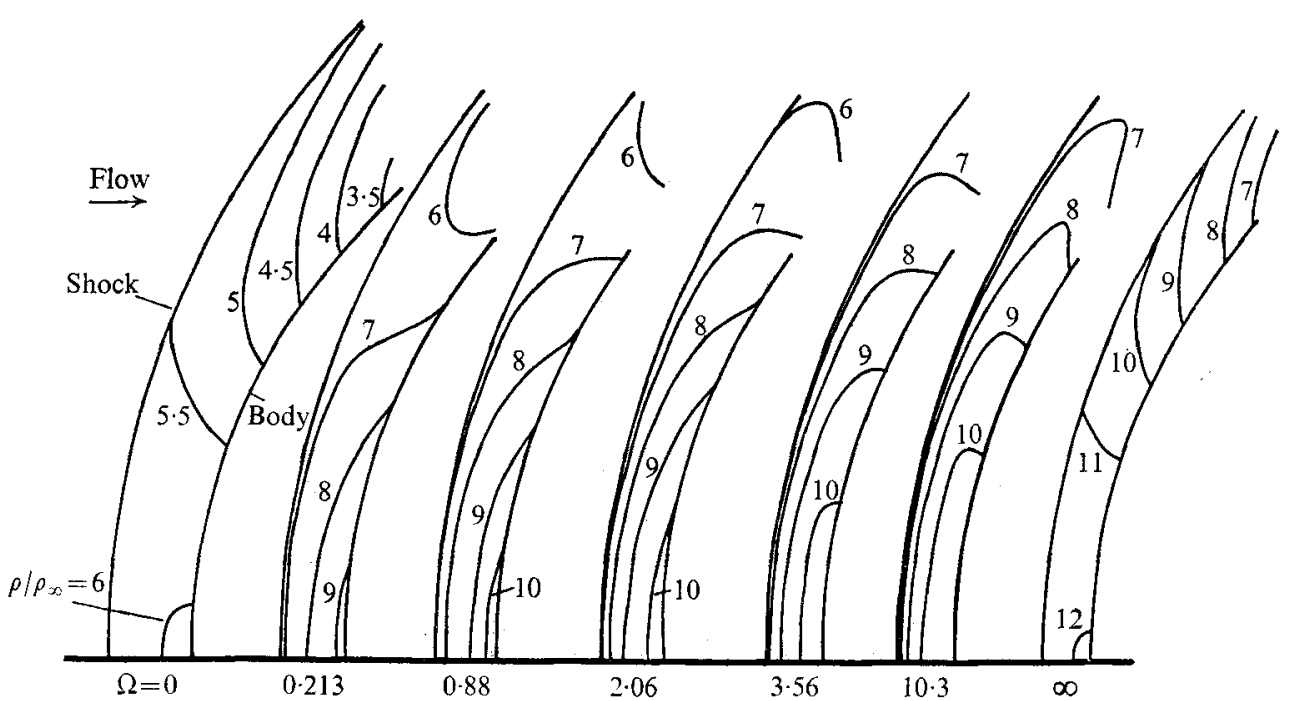

FIgure 8. Density fields for flow over a sphere as calculated by Garr \& Marrone's method except for the cases $\Omega=0$ and $\infty$, which are calculated by Lomax \& Inouye's method.

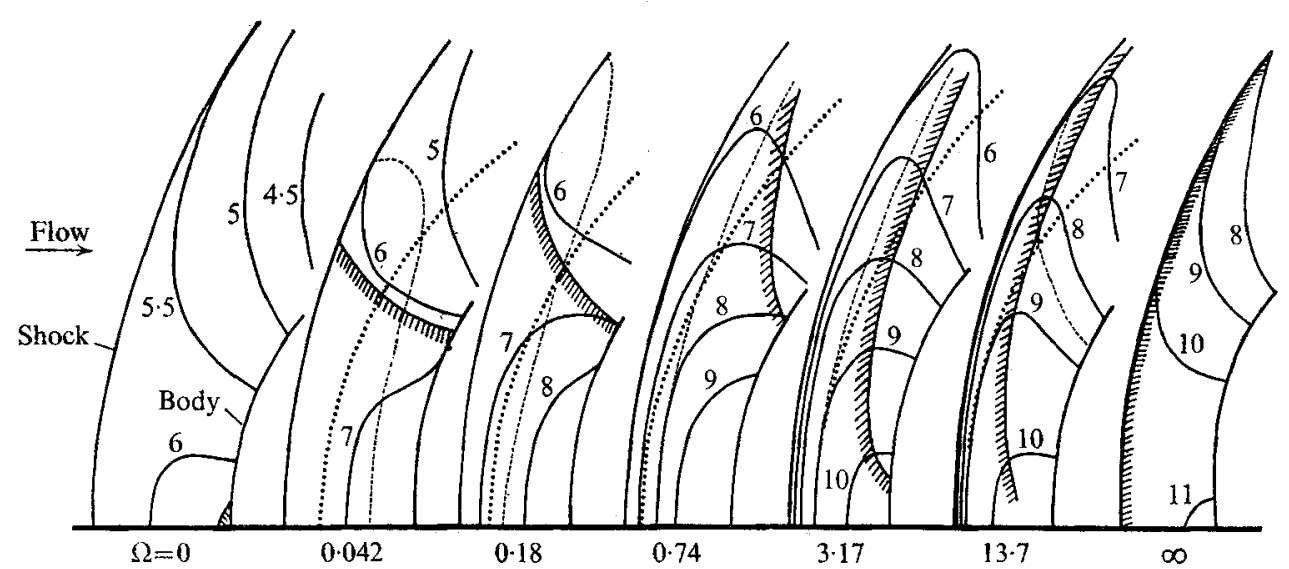

FIGURe 9. Density fields for flow over a cylinder as calculated by Garr \& Marrone's method, with frozen and equilibrium flow as calculated by Lomax \& Inouye's method. - - $\rho / \rho_{\infty}$; $\cdots$, typical $\alpha$ contour; - - , typical temperature contour; w, approximate boundary of region influenced by non-equilibrium dissociation.

frozen and equilibrium flow respectively. Also shown in these diagrams is a boundary between the region where the reaction does not affect the shape of the density contours significantly and the region where the reaction is important. It is seen that as $\Omega$ increaes from zero the region affected by the dissociation grows out of the stagnation point, quickly stretching across to the shock, and then shrinks back into a layer near the shock, finally becoming part of the shock. The similarity between the equilibrium and frozen-flow density patterns is again evident and so also is the manner in which the transition from one to the other 
F

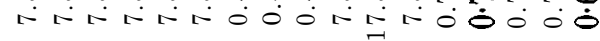

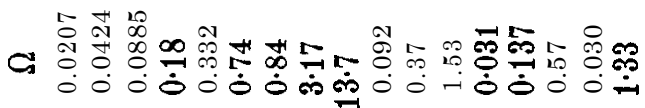

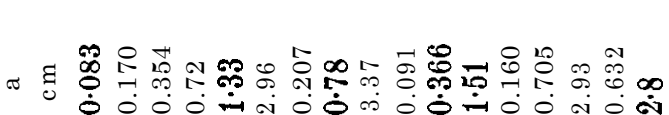

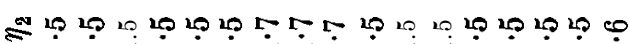
:

$\approx \bar{t}_{0}$

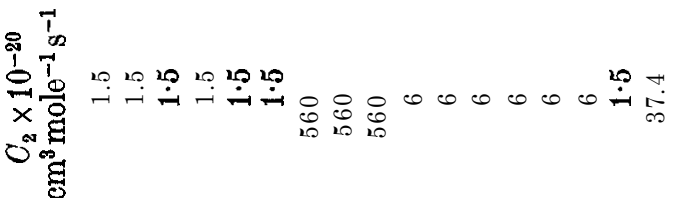

在

$=T^{\top}$

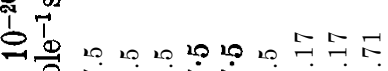

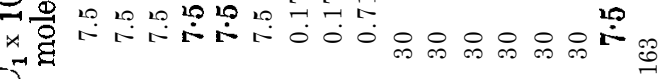
हुํ.

4

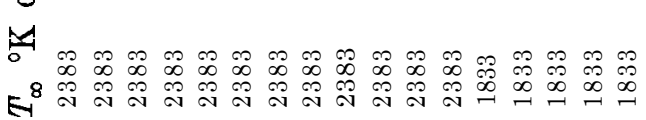
急

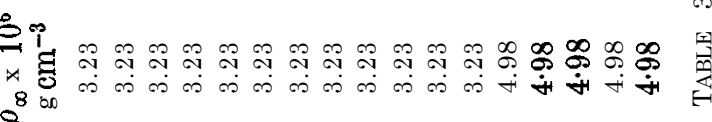
$e^{8}$

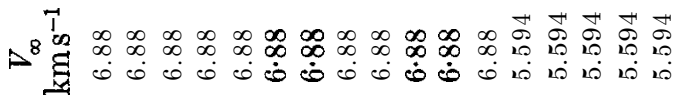

ช 穴 


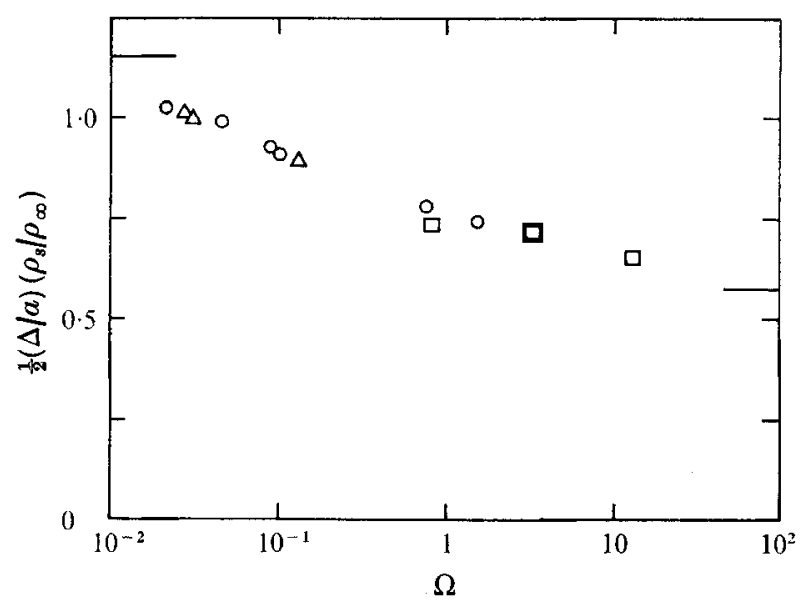

FIGURE 10. Stand-off distance on cylinder calculated by Garr \& Marrone's method. asymptotes calculated by Lomax \& Inouye's method. $O, \alpha_{\infty}=0.424, \Gamma=7 \cdot 36 ; \square$, $\alpha_{\infty}=0.424, \Gamma=0.5 ; \Delta, \alpha_{\infty}=0.07, \Gamma=0.12$.

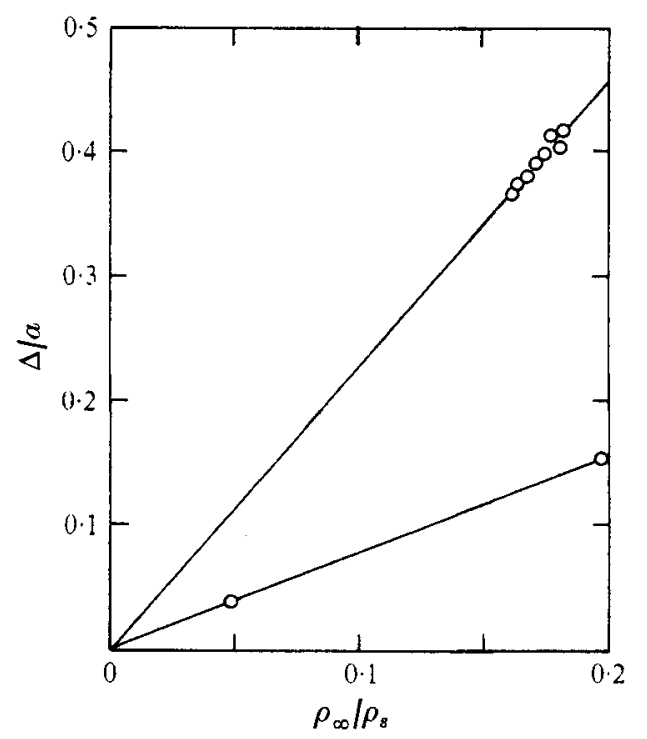

FigURe 11. Stand-off distance for frozen flow over cylinders (upper curve, slope $=2 \cdot 32$ ) and spheres (lower curve, slope $=0.78$ ). $O$, calculation by Lomax \& Inouye's method.

occurs, when the density values on the contours are observed through the range of $\Omega$. Typical contours of each of $\alpha$ and $T$ are superimposed on figure 9 and it is evident that they diverge from each other more than they do in the Freeman model, particularly in regions of weak dissociation. Thus the $\alpha$ and $T$ contours remain more nearly parallel to each other and to the shock layer in the Freeman model because the component of the pressure gradient along streamlines is neglected in the momentum and energy equations.

The conditions at which calculations were made for cylinders are given in table 3 and the stand-off distance $v s . \Omega$ plot for these results is given in figure 10 . 
It is seen that the $\Omega$ correlation holds for the cylinder results in the cases calculated.

In the study of the plane case it became apparent that not many results for the stand-off distance on circular cylinders in frozen or equilibrium flow are available in the literature. The values obtained here by the method of Lomax \& Inouye are therefore plotted against $\rho_{\infty} / \rho_{s}$ in figure 11, together with values for the axisymmetric case. These results, which cover a Mach number range of 6-8 show that the stand-off distance on circular cylinders is mainly dependent on the density ratio as it is for spheres, but that the cylinder stand-off is three times that on spheres.

\section{Experiment}

\subsection{Facility and experimental set-up}

The facility used for all the experiments described here was the large free-pistondriven shock tunnel at A.N.U. known as T3. This device is described in more detail elsewhere (Stalker 1972), but some performance data of the tunnel with nitrogen as the test gas are presented in figure 12. The results in figure 12 are obtained by calculating the nozzle reservoir conditions (subscript 0 ) from the measured shock speed and reservoir pressure, taking the gas to be in thermodynamic equilibrium, and subsequently calculating the non-equilibrium flow through the conical nozzle by the one-dimensional flow method of Lordi, Mates \& Moselle (1966). The temperature on the normal shock is taken from the blunt body calculations by the Garr \& Marrone method for equilibrium vibration and electronic excitation.

It can be seen from figure 12 that the free-stream dissociation rises from zero almost as soon as the reservoir exhibits significant real-gas effects (about $4 \mathrm{~km} / \mathrm{s}$ ) and that for higher speeds $\alpha_{\infty}$ rises approximately linearly with $\mu$. The difference between $\alpha_{0}$ and $\alpha_{\infty}$ is a measure of the energy recovered from dissociation by the nozzle expansion, and $\alpha_{\infty}$ itself is a measure of the degree of non-equilibrium remaining in the free stream, since the free-stream temperature at this area ratio is about $2000^{\circ} \mathrm{K}$ so that the equilibrium value of $\alpha_{\infty}$ is zero. The difference between $T_{0}$ and $T_{s}$ is an approximate measure of the degree of non-equilibrium just behind the normal shock because $T_{s}$ represents the temperature with frozen composition. The importance of understanding the influence of $\alpha_{\infty}$ on the flow over a blunt body is brought out by the behaviour of $\alpha_{\infty}$ with $\mu$. If a flow with an equilibrium free stream $\left(\alpha_{\infty}=0\right)$ is to be simulated in a shock tunnel in which $\alpha_{\infty}$ is 0.4 it must be clear how this modifies the flow field.

To confirm experimentally that the characteristics of the tunnel as calculated (figure 12) are correct, measurements of Pitot pressure, static pressure, shock angle on a wedge and fringe shift across normal and oblique shocks have been made in the facility known as T3. The method of calculating the free-stream conditions was further checked in a prototype facility by approximate massspectrometric measurements of the composition (Slade 1970) and measurements of velocity using spark tracer and magnetohydrodynamic methods (McIntosh 1971). Measurements which involve inserting an instrument into the flow are 


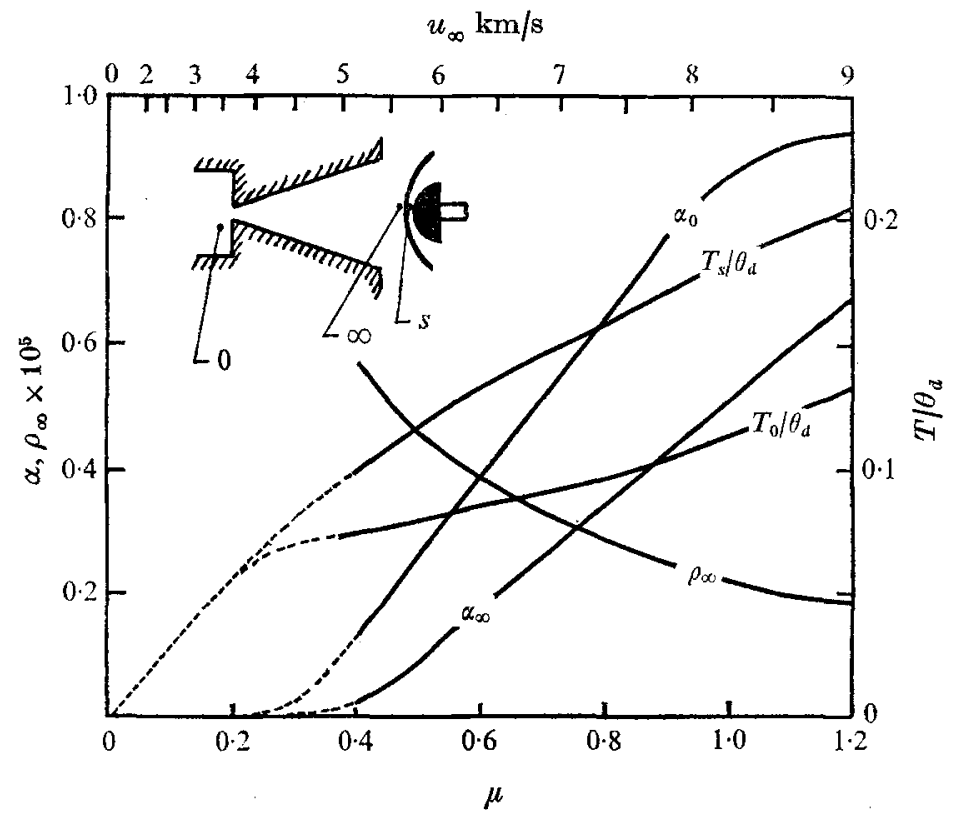

Figure 12. Performance data for T 3 shock tunnel, using nitrogen. Nozzle area ratio $=260$, throat diameter $=0.5$ in., diaphragm burst pressure $=60$, driver compression ratio $=60$.

very difficult at high enthalpy because of the thermal and electrical effects accompanying them. Hence the accuracy of such measurements is not good. To illustrate the accuracy of the experimental confirmation, figure 13 compares the density ratio across a normal shock calculated using the measured fringe shift across a normal shock and the calculated free-stream density with the more precise form of Freeman's expression for the density ratio,

$$
\frac{\rho_{s}}{\rho_{\infty}}=\frac{7+\alpha_{\infty}}{4+\alpha_{\infty}}\left\{1-\left(1-\frac{\left[\mu+\left(4+\alpha_{\infty}\right) T_{\infty} / \theta_{d}\right]\left(1+\alpha_{\infty}\right)\left(7+\alpha_{\infty}\right)}{\mu\left(4+\alpha_{\infty}\right)^{2}}\right)^{\frac{1}{2}}\right\}^{-1},
$$

which assumes the vibrational degrees of freedom of the molecules to be half excited, as well as with the density ratio expected from frozen composition and equilibrium vibration. Equation (27) differs from (17) in that the contribution to the total enthalpy from the normal component of velocity has not been neglected at the shock (as it has in (14)). More accurate experimental results for the density ratio can be obtained by measuring the shock angle on a wedge. In non-equilibrium flow the wedge shock is curved, but if the wedge angle is sufficiently shallow the shock is straight. Source-flow effects also curve the shock, but a calculation according to the method of Hall (1963) shows that the contribution to shock curvature is typically $0.005 \mathrm{~cm}^{-1}$ for a $35^{\circ}$ wedge at $80 \mathrm{~cm}$ from the source. Under the conditions of the present experiments the shock wave on a $35^{\circ}$ wedge does not raise the temperature sufficiently to cause a significant reaction rate and the shock is straight (frozen flow). Results obtained on a $35^{\circ}$ wedge are presented in figure 13. The experimental results lie between the curves for halfexcited and fully excited vibration and well below the curve obtained in the 


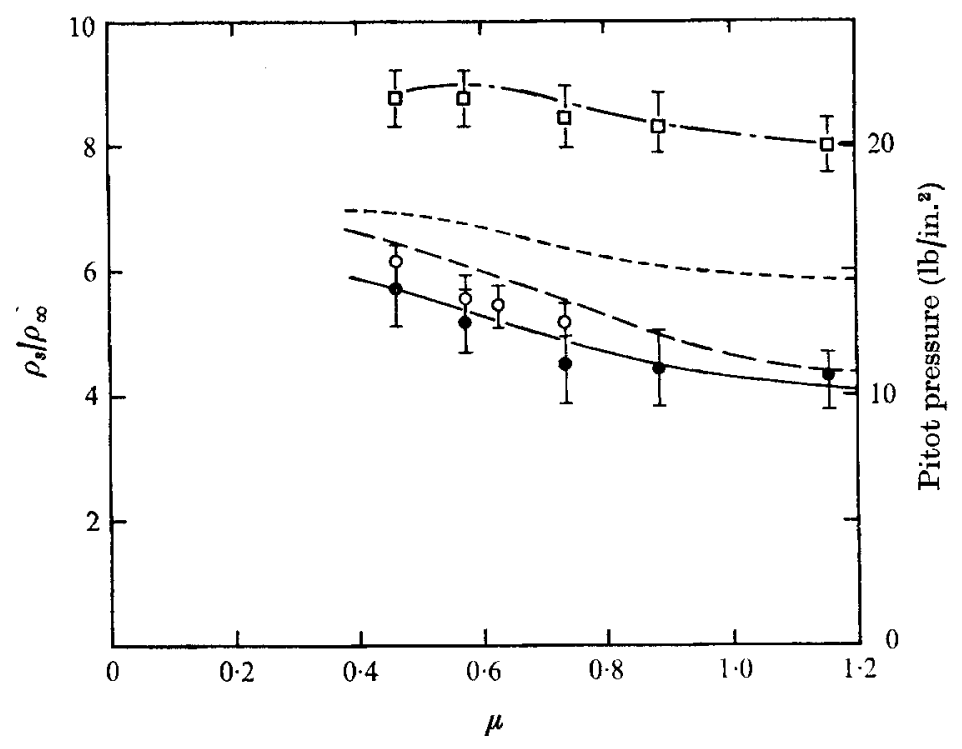

FIGURE 13. Density ratio across a normal shock and Pitot pressure in T3. Values of $\rho_{8} / \rho_{\infty}$ : $\Phi$, from shock angle on $35^{\circ}$ wedge; $\Phi$, from fringe shift and calculated density; - , vibration half excited (equation 25 ); $-\ldots$-, equilibrium vibration; $\cdots$, , equilibrium vibration and electronic excitation. Pitot pressure: - - _ calculated, 兵, measured.

Garr \& Marrone calculations for equilibrium vibration and electronic excitation. Because of the source-flow effect the free-stream density varies with axial position along the nozzle. This also affects the density ratio obtained from fringe shift and calculated density (filled symbols in figure 13). However, the density ratio as obtained from the wedge shock depends only on wedge and shock angles. The open circles in figure 13 represent the density ratio across an equivalent normal shock at the calculated free-stream Mach number.

Also shown in figure 13 are the measured and calculated values of the Pitot pressure. Since the nozzle is conically divergent (at $15^{\circ}$ included angle) the Pitot pressure is sensitive to the axial position of the probe. To decide where the effective nozzle area ratio is equal to the area ratio of the nozzle-flow calculation, the calculated and experimental Pitot pressures are matched at the low enthalpy end $(\mu=0.46)$, and the remaining nozzle-flow calculations are made to the same area ratio. This procedure indicates a positive displacement thickness of the nozzle-wall boundary layer. The approximate agreement of the two methods of determining the density ratio confirm the value of the free-stream density to an accuracy of $\pm 10 \%$. The value of the density ratio indicated can be used to determine the free-stream dissociation fraction by assuming that the excitation of the vibrational degree of freedom of the gas is increased, as it passes across the shock, by $75 \%$ of its maximum value. An estimate of the error in the value of $\alpha_{\infty}$ obtained in this manner is \pm 0.07 . The Pitot pressure can then be used to calculate the free-stream speed to an accuracy of $\pm 10 \%$. All the calculated values are found to fall within the estimated error bands. 
The interpretation of an interferogram is easiest if the flow field to be studied is plane. This also gives a larger optical path than an axisymmetric flow field. Accordingly the model chosen is a circular cylinder in a cross fow. It can be seen from figure 12 that the parameters $\mu$ and $\alpha_{\infty}$ cannot be varied independently in the facility. However, since $\alpha_{\infty}$ is a particularly important parameter it is chosen in suitable intervals at $\alpha_{\infty}=0 \cdot 07,0 \cdot 14,0 \cdot 27,0.43$ and 0.66 , corresponding to $\mu=0.46,0.57,0.73,0.88$ and 1.16 respectively. The reaction rate parameter can be varied at a given tunnel condition by adjusting the diameter of the model. This has an upper limit of about $4 \mathrm{in}$. because of the useful diameter of the flow (about $6 \mathrm{in}$. with actual diameter $8 \mathrm{in}$.), and a lower limit of about $\frac{1}{8}$ in. because of the resolution of the optical system. The models chosen were thus a set of cylinders 6 in. long with diameters $\frac{1}{8}, \frac{1}{4}, \frac{1}{2}, 1,2$ and $4 \mathrm{in}$. The range of the reaction rate parameter which can be achieved with these conditions and models covers $2 \frac{1}{2}$ decades.

In the experiment, reservoir pressure and shock speed are monitored, defining the reservoir conditions for the nozzle, and the Pitot pressure is monitored in the test section. The shock speed is measured by measuring the time intervals between five consecutive stations along the shock tube in which the arrival of the shock is indicated by the jump in electrical conductivity of the gas, which is sensed by a sudden current passing between two small probes set back in a tapping on the shock tube wall. The reservoir pressure is measured with a Kistler Type 6201 quartz-crystal pressure transducer and is about $220 \mathrm{~atm}$. The Pitot pressure is measured with a Kistler type $603 \mathrm{H}$ miniature acceleration-compensated quartz-crystal pressure transducer housed in a brass mounting, with a $1 \mathrm{~mm}$ diameter brass tube, $2 \mathrm{~cm}$ long, acting as the Pitot tube and connected to the small volume on the face of the pressure transducer. Figure 14 (plate 1) shows a typical record of the reservoir and Pitot pressure traces in which the time base is $200 \mu$ s per major division. It can be seen that the Pitot pressure is approximately constant for $500 \mu \mathrm{s}$. The large break in the Pitot pressure trace is caused by the electrical noise from the exploding-wire light source which is used for taking interferograms.

The Mach-Zehnder interferometer used has an $8 \mathrm{in}$. field of view and can be set either for infinite or finite fringe width, depending on the conditions and purpose of the particular experiment. The light source is an exploding wire $0.3 \mathrm{~mm}$ in diameter through which a capacitor of $500 \mu \mathrm{F}$ at $4.5 \mathrm{kV}$ is discharged. This produces a bright flash for about $100 \mu \mathrm{s}$ which is intense enough to swamp the strong gas luminosity in the flow field. A band-pass filter of $4300 \pm 100 \AA$ introduced just before the camera lens ensures that a large number of fringes are of good contrast. In order to produce a parallel beam of light through the test section, an image of the exploding wire is focused on a small aperture $\left(\frac{1}{2} \mathrm{~mm}\right.$ diameter) at the focal point of the input lens of the interferometer. An image of this source is formed by the interferometer output lens, and a stop just accommodating the image of the light source is introduced there also, to reduce the amount of light passing through to the camera from the flow luminosity. 


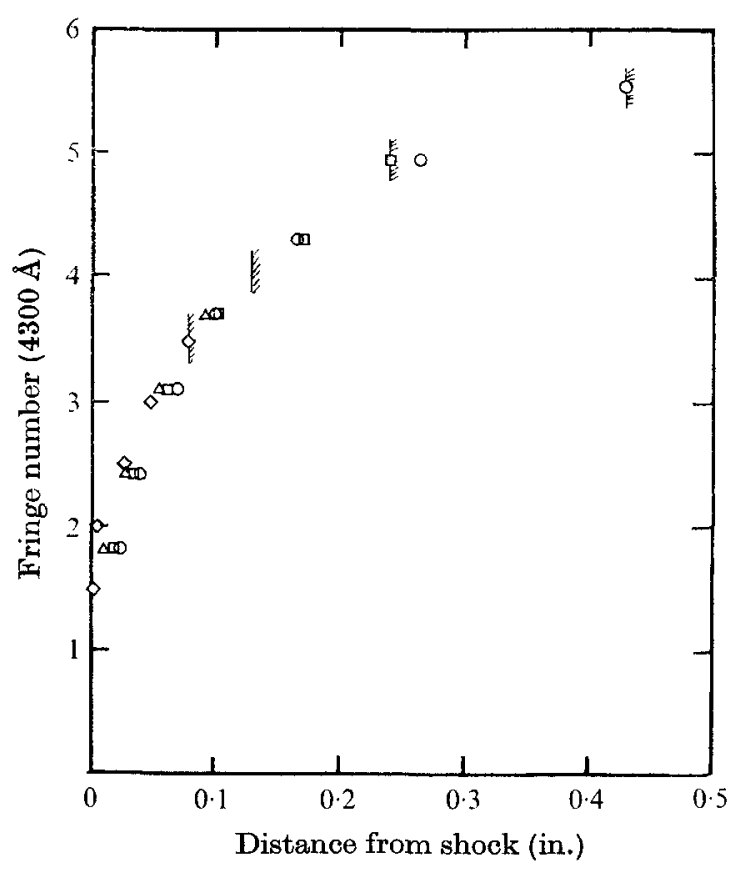

FraURE 16. Fringe shift relative to free stream along the stagnation streamline of a circular cylinder, $\mu=0.46, \alpha_{\infty}=0.07$. (The fringe number of photographs taken at $\lambda=5300 \AA$ is converted to the equivalent at $\lambda=4300 \AA$.) Cylinder diameter: $O, 4$ in.; $\square, 2$ in.; $\Delta, 1$ in.; $\diamond, \frac{1}{2}$ in. E, edge of body.

\subsection{The fringe pattern}

The interferograms obtained at $\mu=0 \cdot 46$ are presented in figure 15 (plate 2). All but the case of the half-inch diameter model are taken with infinite fringe width, so that the fringes represent contours of optical path. The fringe shift $F$ is related to the density change $\Delta \rho$ by

$$
\Delta \rho=\frac{4 \cdot 16 F \lambda}{L(1+0 \cdot 28 \alpha)} \mathrm{g} \mathrm{cm}^{-3},
$$

where $\lambda$ is the wavelength and $L$ is the geometrical path. The dissociation fraction appears in (28) because the refractivity of atomie nitrogen is higher than that of molecular nitrogen (see Alpher \& White 1959). The fringe pattern thus gives a qualitative picture of the density pattern provided that $\alpha$ does not change too much. The error is less than $10 \%$ if $\alpha$ varies by 0.35 . Measurements of fringe shift over a wide range of wavelengths (channelled spectra) indicate that the contribution to the refractive index due to the presence of electrons in the gas is not significant in the present experimental conditions (Sandeman, private communication). Note that the wavelength is not the same for all the photographs of figure 15.

The fringe patterns illustrate the features of figure 9 fairly well. At the high $\Omega$ end ( $4 \mathrm{in}$. diameter) the fringe number on the stagnation streamline rises rapidly from the shock and flattens off towards the body, while the fringes behave more 


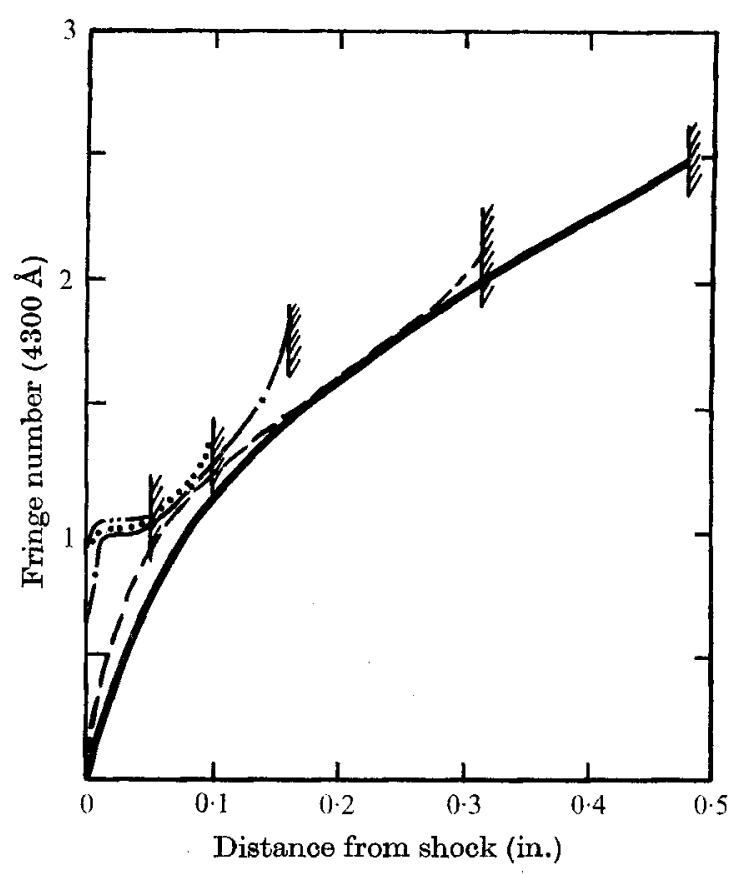

Figure 18. Fringe shift relative to free stream along stagnation streamline of circular cylinder, $\mu=0.73, \alpha_{\infty}=0.27, \lambda=4300 \AA$. Cylinder diameter: - , 4 in.;,--- 2 in.; - - - 1 in.; $\ldots, 0.5$ in.; _ - . - 0.25 in. E, body.

like the pressure contours at the side of the eylinder. The latter feature is more easily seen on the $2 \mathrm{in}$. diameter model, which still exhibits the strong rise of fringe number towards the stagnation point. This is reduced considerably on the 1 in. and $\frac{1}{2}$ in. diameter models. An interesting feature on the 2 in. diameter model is that the boundary-layer separation on the rear of the model can be seen quite clearly. The fringe number on the stagnation streamline is plotted against distance from the shock for these cases in figure 16, which shows that the fringe shift basically follows the same curve, and is only cut off by the body in different positions for the different body sizes.

Finite fringe interferograms of the flow at $\mu=0.73$ are presented in figure 17 (plate 3) and the fringe number along the stagnation streamline is again shown in figure 18. Both figures once more illustrate the same features, but the fringe shift close to the shock is seen to be dependent on the body size. This feature is due to the end effect on the large cylinders, which causes the shock to be convex in a lateral direction and thus the detailed features of the fringe shift near the shock to become smeared out for the 2 in. and 4 in. diameter cylinders, for which the length-to-diameter ratios are only 1.5 and 3. A very interesting phenomenon appears, however, in the smaller bodies. The fringe shift hesitates after the shock for a short distance and then rises, steeply at first and flattening out further downstream. The hesitation is consistent with an induction time for nitrogen dissociation as predicted by Shui, Appleton \& Keck (1970) on the basis of theoretical calculations. According to these results dissociation occurs at 


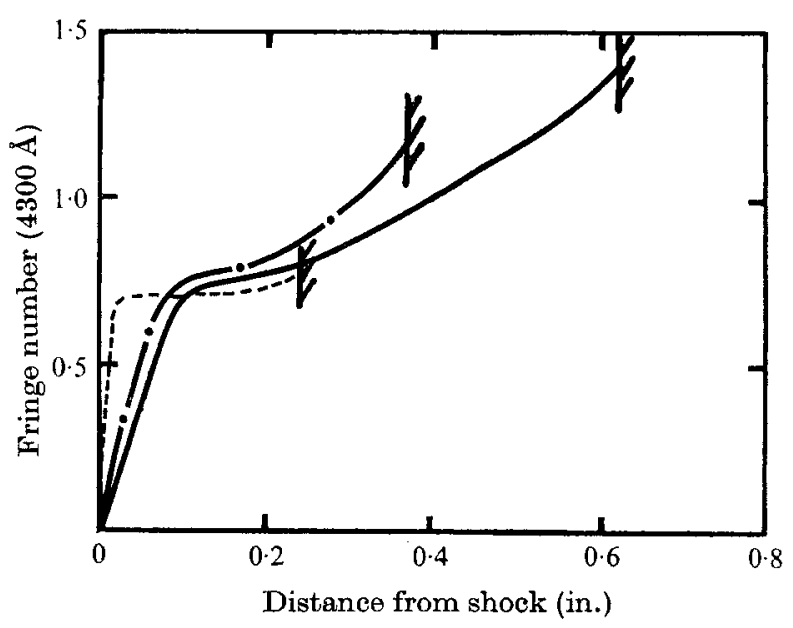

Figure 19. Fringe shift relative to free stream along stagnation streamline of cylinder, $\mu=0.88, \alpha_{\infty}=0.66, \lambda=4300 \AA$. Cylinder diameter: —_, 4 in.; - - , 2 in.; - - 1 in.

significant rates only after the electronically excited $A^{3} \Sigma_{u}^{++}$state of the nitrogen molecule is populated. This feature is more markedly exhibited by the results at $\mu=0.88$, which are plotted in figure 19 . The behaviour of this induction distance with the ambient conditions has been measured from these and other experimental results and compared favourably with the form predicted by Shui et al. (1970). These results are reported in Hornung (1972). The induction distance cannot be detected in the results at $\mu=0 \cdot 46$, mainly because the induction time becomes quite small as the molecular nitrogen concentration increases. However, an experiment was made at approximately this condition $\left(\mu=0.46, \alpha_{\infty}=0.07\right)$ but at a density lower by a factor of four than that given in figure 12; the resulting finite fringe interferogram (figure 20, plate 4 ) shows the induction distance quite clearly. The induction time is much larger than the time for vibrational relaxation as given by Millikan \& White (1963). The latter would correspond to a distance after the normal shock of about $0.5 \mathrm{~mm}$ in the present conditions.

This phenomenon can be expected to modify the results of the theory considerably, particularly at high $\mu$ where the induction distance is larger. Thus the reaction rate parameter $\Omega$ can no longer be expected to correlate the stand-off distance or density pattern at the high enthalpies and at the small body dimensions, but it should be satisfactory at lower enthalpies.

Unfortunately, the distortion of the density pattern with the 2 and 4 in. diameter models due to three-dimensional effects probably affects the behaviour of the fringe shift near the stagnation point as well as near the shock, because the geometric path through the shock envelope is longer than 6 in. at the stagnation point, becoming shorter and shorter towards the shock. This can be expected to distort the pattern in such a way as to raise the fringe number near the stagnation point and to lower it near the shock. The latter effect is evident in figure 16 , in which the fringe shift near the shock is seen to decrease as the body size is increased. 


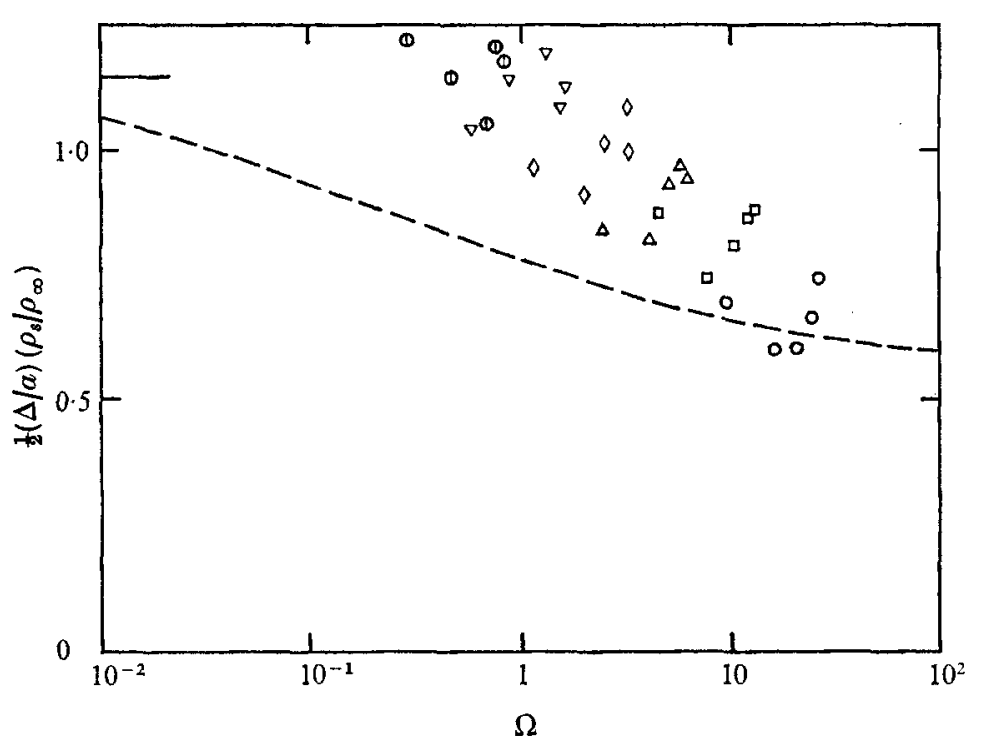

FIGURE 21. Measured stand-off distance on circular cylinders. -- , theory (figure 10); $\longrightarrow$, asymptote for $\Omega \rightarrow 0$. Cylinder diameter: $O, 4$ in.; $\square, 2$ in.; $\triangle, 1$ in.; $\diamond, \frac{1}{2}$ in.; $\nabla, \frac{1}{4}$ in.; (1), $\frac{1}{8}$ in.

In all the interferograms the boundary layer is visible as a sharp rise in the fringe shift near the wall. Near the stagnation point this density rise can be expected to cause the displacement thickness of the boundary layer to be much smaller than the boundary-layer thickness, from the results of Scala \& Baulknight $(1960$, figure 1), who also found that the thickness indicated by the density profile corresponds approximately to that of the velocity profile. Thus the effect of the boundary-layer displacement thickness on the stand-off distance may be neglected. The Reynolds number, based on a length of $1 \mathrm{~cm}$, behind the normal shock is typically $1.5 \times 10^{3}$.

\subsection{The stand-off distance}

For the purposes of presenting the experimental results on the stand-off distance, the reaction rate prameter $\Omega$ was calculated from the dissociation rates given by Cary (1965), extrapolated to the temperature of the experiment according to Cary's recommended formula. The stand-off distance parameter is calculated using $\rho_{s} / \rho_{\infty}$ as given by a mean line through the values obtained from measurements of the shock angle on a $35^{\circ}$ wedge (see figure 13). The results are shown in figure 21 together with a curve which represents a mean line through the theoretical results of figure 10. Two things are apparent immediately. First, the results follow a much steeper slope than is expected from theory and lie well to the right of it. Second, they exhibit an effect which is connected systematically with the model size and with the tunnel condition. For each of the four large model sizes the curve goes through a characteristic dip at $\alpha_{\infty}=0 \cdot 27$, rising steeply towards the high enthalpy end (increasing $\Omega$ ). For the two smaller model sizes ( $\frac{1}{4}$ and $\frac{1}{8}$ in. diameter) this behaviour is lost in the experimental error of measuring the stand-off distance. 


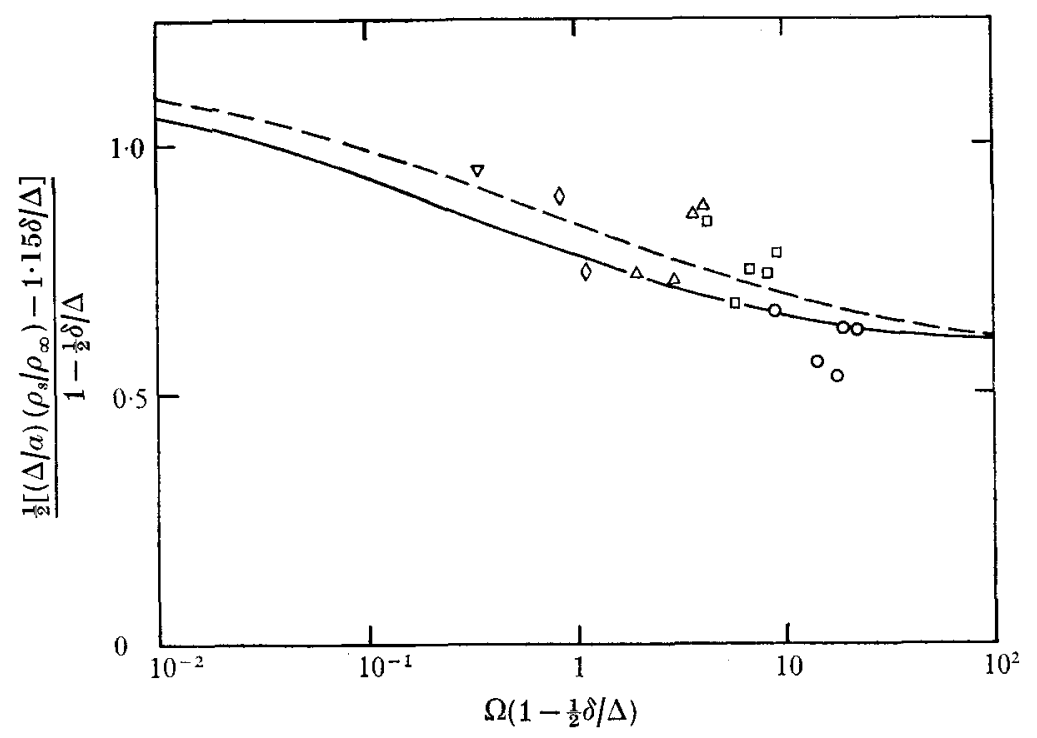

FIGURE 22. Stand-off distance on circular cylinder, adjusted for induction effect. - - theory (figure 10); ---, same curve displaced to the right by a factor of $2 \cdot 5$. Cylinder diameter: $O, 4$ in.; $\square, 2$ in.; $\Delta, 1$ in.; $\diamond, \frac{1}{2}$ in.; $\nabla, t$ in.

An effect such as this may be expected from the induction time phenomenon, which keeps the density at a low value for a larger proportion of the shock-layer thickness on smaller bodies, where the stand-off distance may thus be expected to be higher than the theoretical curve. The measured induction distances also follow this characteristic dip and rise to a value of $4 \mathrm{~mm}$ at $\alpha_{\infty}=0.65$ (see Hornung 1972). In an attempt to correct for the effect of induction distance, the stand-off parameter is plotted in the form

$$
\left.\frac{\frac{1}{2}\left[(\Delta / a)\left(\rho_{s} / \rho_{\infty}\right)-1 \cdot 15 \delta / \Delta\right]}{1-\frac{1}{2} \delta / \Delta} \text { vs. } \Omega\left(1-\frac{1}{2}\right) \delta / \Delta\right)
$$

in figure 22. Here $\delta$ is the measured induction distance. This form is chosen such that at small $\delta / \Delta$ a quantity proportional to $\delta / \Delta$ is subtracted from the stand-off parameter and when $\delta / \Delta$ approaches one (and thus when the stand-off parameter $\frac{1}{2}(\Delta / a)\left(\rho_{s} / \rho_{\infty}\right)$ approaches $\left.1 \cdot 15\right)$ the corrected form also approaches $1 \cdot 15$. The reaction rate parameter is modified because the distance available for the dissociation process between body and shock is reduced by the induction distance. It can be seen that this crude adjustment removes the systematic departure of the experimental points from their mean and brings the slope of the results more into line with the expected behaviour.

It should be noted that the points for which $\delta / \Delta>1$ were omitted in figure 22 . By referring back to figure 21 it can be seen that all the points omitted in figure 22 seatter around the frozen-flow asymptote of $\Delta \rho_{s} / 2 a \rho_{\infty}=1.15$ with a maximum error of $\pm 10 \%$. This corresponds to about $\pm 0.005 \mathrm{in}$. in the $\frac{1}{4} \mathrm{in}$. diameter model and is near the limit of resolution of the optical system and alignment acouracy. The accuracy of the experimental results is also limited at low $\Omega$ by the induction 
distance and at high $\Omega$ by the three-dimensional effect of the flow over the $4 \mathrm{in}$. diameter cylinder. The three-dimensional effect manifests itself slightly in a lowering of the stand-off distance below the asymptotic equilibrium value on the large model. A further limitation on the accuracy is the uncertainty in the density ratio across the normal shock which is part of the stand-off parameter (see figure 13).

\subsection{The reaction rate}

The reaction rate data given by Cary yield composite reaction rates about 2-3 times higher than the more recent results of Appleton, Steinberg \& Liquornik (1968), which were obtained at temperatures up to $15000^{\circ} \mathrm{K}$. Thus the theoretical curve in figure 22 has been shown dotted and displaced to the right by a factor of 2.5. It can be seen that the quality of the experimental results is not good enough to distinguish between these two recommended reaction rates.

A more sensitive way of estimating the reaction rate is shown in figure 23 (plate 4), which compares the calculated density pattern with the interferogram for the case of $\mu=0 \cdot 4, \alpha_{\infty}=0.07$ and $\Omega=0.57$. By calculating the flow pattern for different values of reaction rate, it is considered possible to estimate $\Omega$ to within a factor of two by this method. However, this does not give a satisfactory measurement of the constants $C_{1}$ and $C_{2}$ since the relation between these and $\Omega$ involves a very strong function of temperature and the temperature is not known sufficiently accurately. The overall trend of the experimental results suggests, however, that the dissociation rate is somewhat lower than that recommended by Cary.

\section{Conclusions}

The results of the theoretical calculations show that the dissociating flow of nitrogen on the front part of a blunt body can be approximately correlated in terms of a single reaction rate parameter $\Omega$, taking account of the effects of all the relevant parameters. As has been observed before, the pressure field is insensitive to $\Omega$. The correlation applies to the flow pattern as well as to the standoff distance. The density field, which is sensitive to the reaction rate, can be roughly divided into two regions: that dominated by the reaction, where density contours follow contours of dissociation fraction, and that dominated by the pressure field, where the density contours follow the pressure contours. The relative size of these regions and the boundary between them are described in terms of $\Omega$.

Two conclusions may be drawn from these results. First, the correlation allows flows, in which the free-stream dissociation is zero, to be simulated in a facility where $\alpha_{\infty}$ is finite. Second, the qualitative picture of the transition from a pressure-dominated to a reaction-dominated region (figure 9) suggests that a considerable simplification of the theoretical model of reacting flow may be possible, separately in the two regions, with a suitable matching between them.

The experimental results illustrate clearly that reacting nitrogen flows can be achieved in a free-piston-driven shock tunnel. The results agree qualitatively with numerical computations, but exhibit a very strong scale effect. This is 
found to be consistent with an induction time for nitrogen dissociation as suggested by Shui, Appleton \& Keck (1970). This unexpected feature illustrates the importance of doing the experiment rather than relying entirely on numerical results in studying high enthalpy flow problems.

The experimental results can be used to estimate the effective reaction rate parameter $\Omega$ to within a factor of two but, because of the uncertainty in the temperature, do not give reliable reaction rate constants.

The construction and maintenance of the shock tunnel used in the above experiments was financed by a grant from the Australian Research Grants Committee, whose support is gratefully acknowledged. I would also like to thank Mr R. French for his technical advice and assistance.

\section{REFERENCES}

AlPher, R. A. \& WhIte, D. R. 1959 Phys. Fluids, 2, 162.

Appleton, J. P., Stetingera, M. \& Liquornik, D. J. 1968 J. Chem. Phys. 48, 599.

Belotserkovski, O. M. \& DUShin, V. K. 1964 J. Chem. Mech. Math. Phys. 4, 61.

CARY, B. 1965 Phys. Fluids, 8, 26.

Freeman, N. C. 1958 J. Fluid Mech. 4, 407.

Garr, L. J. \& Marrone, P. V. 1963 Cornell Aeron. Lab. Rep. QM-1626-A-12(II).

HaLl, J. G. 1963 Cornell Aeron. Lab. Rep. no. 128.

Hornung, H. G. 1971 A.I.A.A. J. 9, 1198.

Hornung, H. G. $1972 J$. Chem. Phys. 56, to appear.

Kyriss, C. L. 1970 A.I.A.A. Paper, 70-771.

LICK, W. 1960 J. Fluid Mech. 7, 128.

LIGHTHILL, M. J. 1957 J. Fluid Mech. 2, 1.

Lomax, H. \& Inouye, M. 1964 N.A.S.A. Tech. Rep. R-104.

Lordi, J. A., Mates, R. E. \& Moselde, J. R. 1966 N.A.S.A. Current Rep. CR-472.

McLstosm, M. K. 1971 Phys. Fluids, 14, 1100.

Millikan, R. C. \& White, D. R. 1963 J. Chem. Phys. 39, 98.

Scala, S. M. \& BaUlknight, C. W. 1960 A.R.S. J. 30, 329.

Shut, V. H., Aprleton, J. P. \& KeCK, J. C. 1970 J. Chem. Phys. 53, 2547.

SLADE, J. C. 1970 M.Sc. thesis, Australian National University, Canberra.

SPURK, J. H. 1970 A.I.A.A.J. 8, 1039.

SPURK, J. H. \& BARTOs, J. M. 1966 Phys. Fluids, 9, 1278.

Stalker, R. J. 1972 Aeron. J. to be published. 


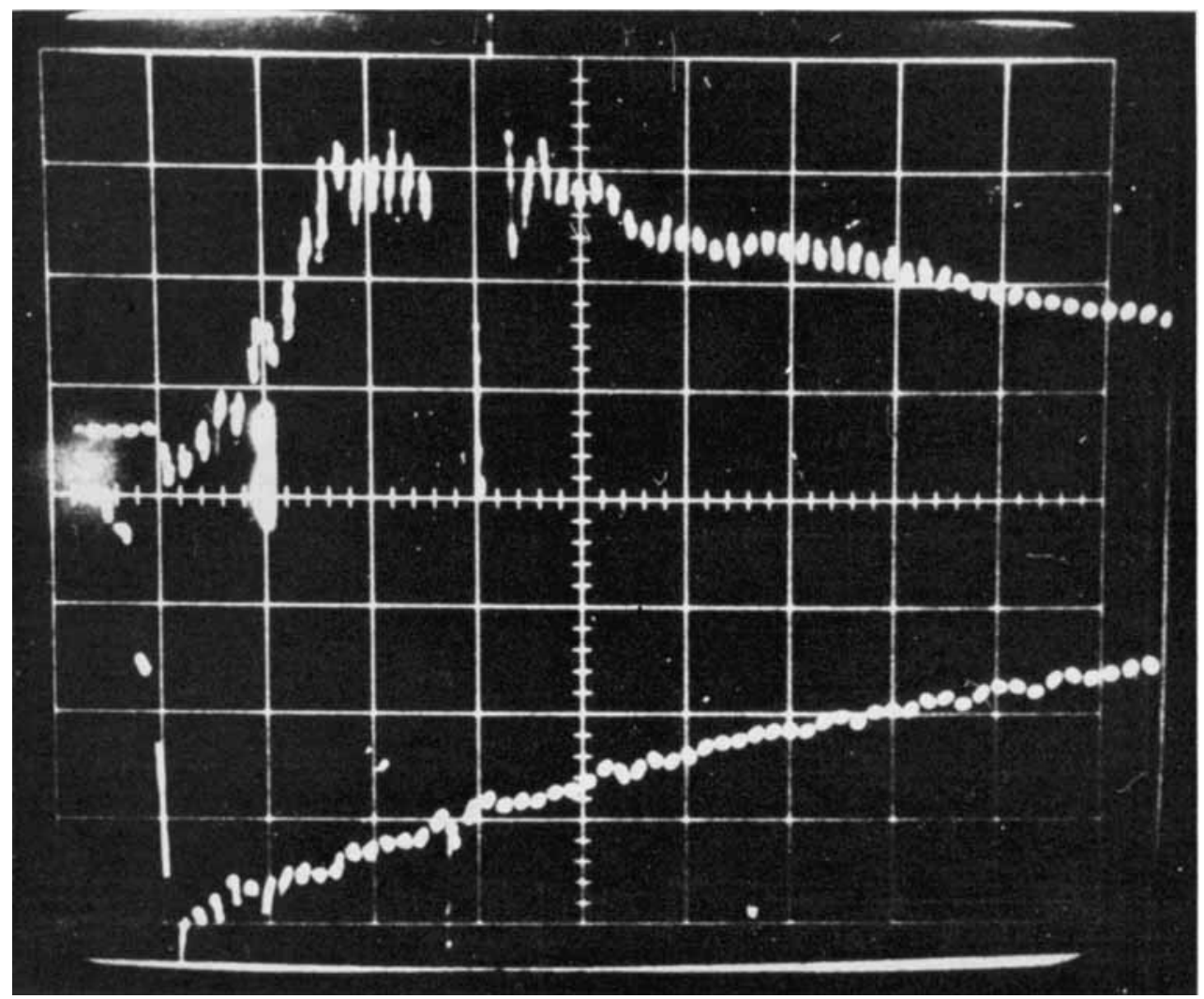

FIGURE 14. Reservoir and Pitot pressure traces, $\mu=\mathbf{0 \cdot 4 6}$. Upper trace, Pitot pressure; lower trace, reservoir pressure (measuring positive downward). Time base: $200 \mu \mathrm{s}$ per major division. 


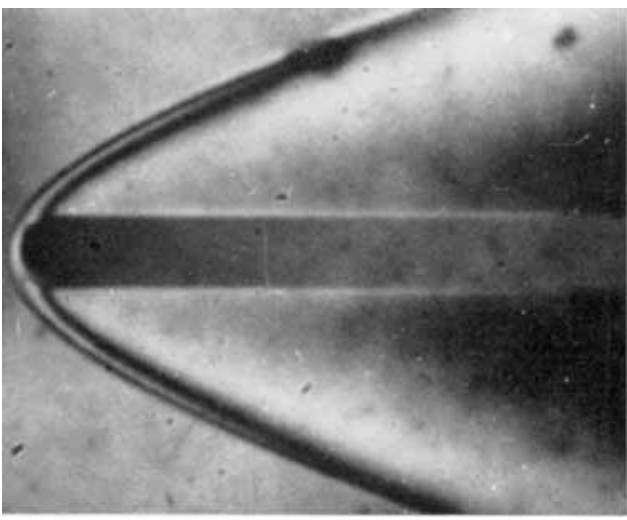

(a)

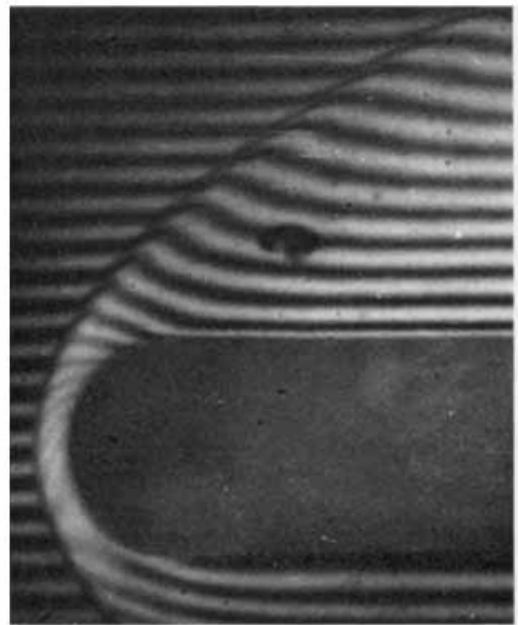

(c)

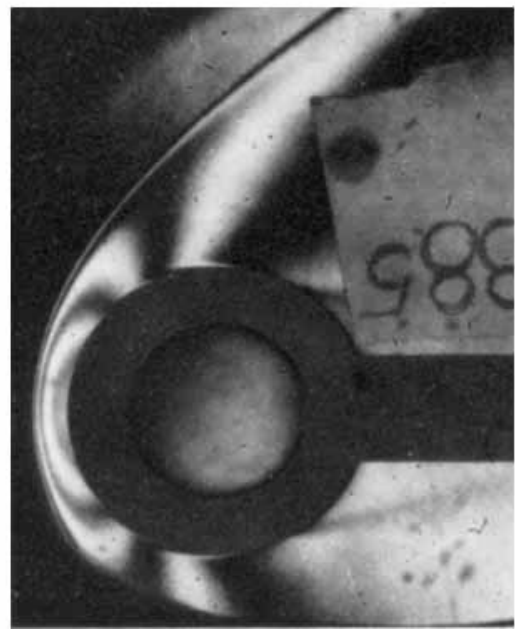

(e)

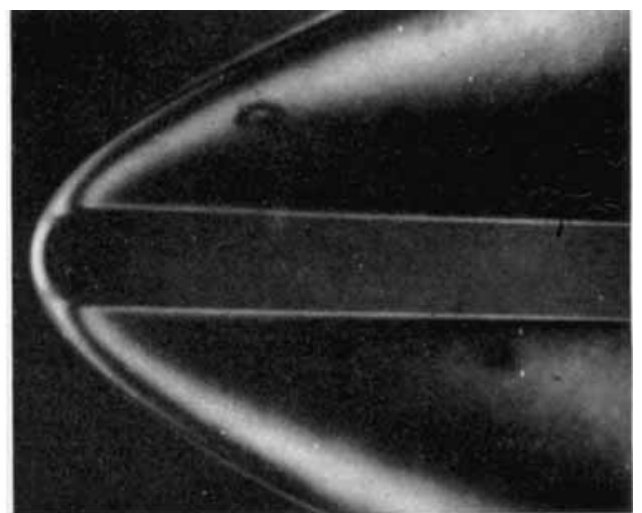

(b)

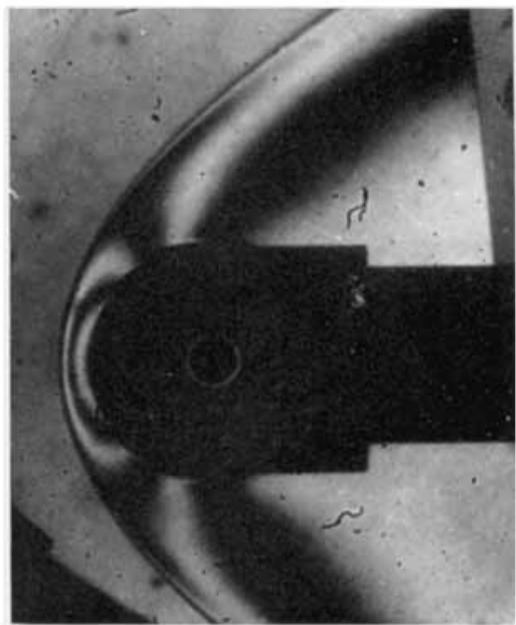

(d)

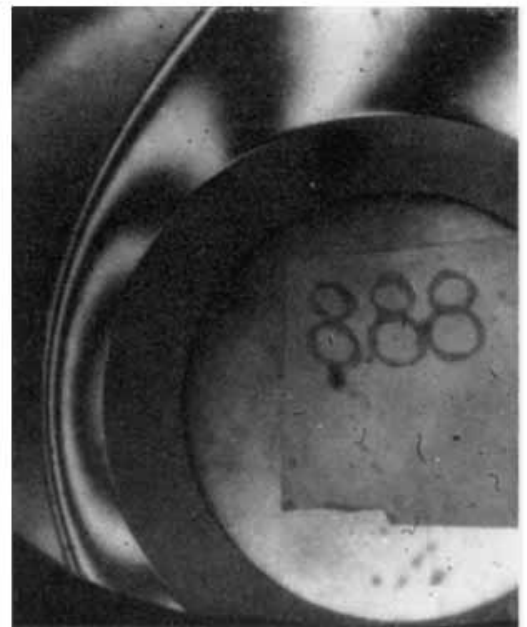

(f)

FigUre 15. Interferograms of flow over circular cylinder at $\mu=0 \cdot 46, \alpha_{\infty}=0 \cdot 07$. (a) $0.125 \mathrm{in}$. diameter, $\lambda=4300 \AA$, infinite fringe. (b) $0.25 \mathrm{in}$. diameter, $\lambda=4300 \AA$, infinite fringe. (c) $0.5 \mathrm{in}$. diameter, $\lambda=4300 \AA$, finite fringe. $(d) 1.0 \mathrm{in}$. diameter, $\lambda=5330 \AA$, infinite fringe. (e) $2 \cdot 0 \mathrm{in}$. diameter, $\lambda=5330 \AA$, infinite fringe. $(f) 4 \cdot 0 \mathrm{in}$. diameter, $\lambda=5330 \AA$, infinite fringe. 


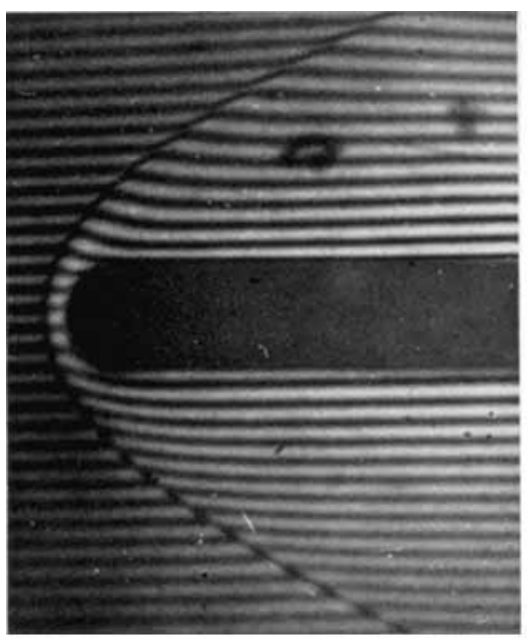

(a)

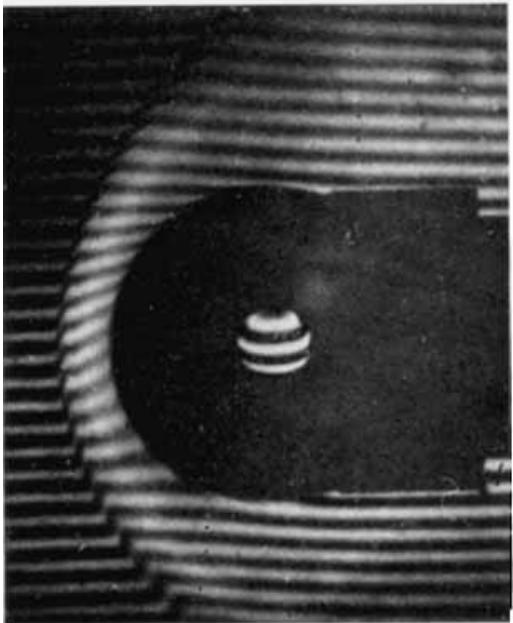

(c)

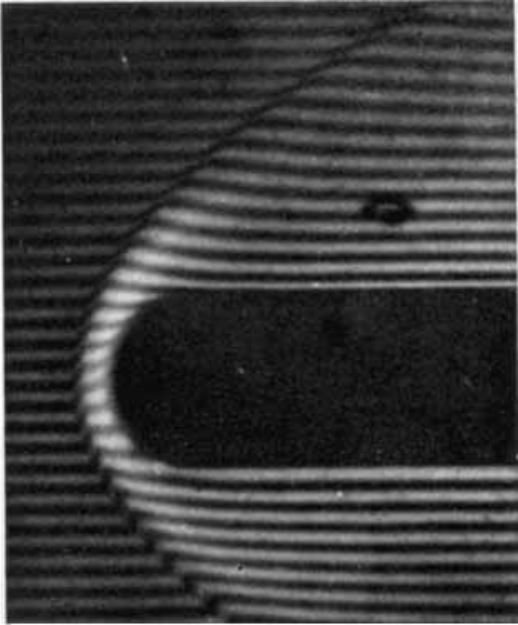

(b)

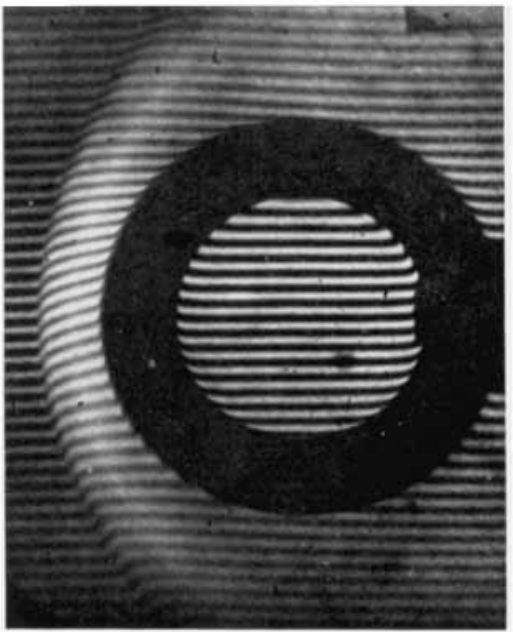

(d)

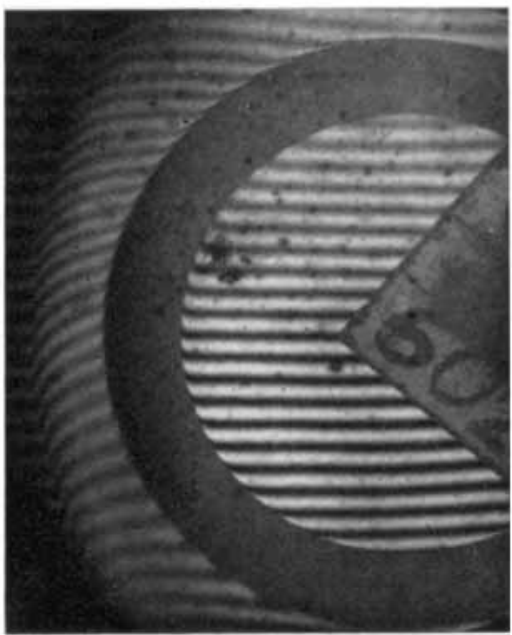

(e)

Figure.17. Interferograms of flow over circular cylinder at $\mu=0 \cdot 73, \alpha_{\infty}=0 \cdot 27, \lambda=4300 \AA$. (a) 0.25 in. diameter. $(b) 0.5$ in. diameter. $(c) 1.0$ in. diameter. $(d) 2.0 \mathrm{in}$. diameter. $(e) 4.0 \mathrm{in}$. diameter. 


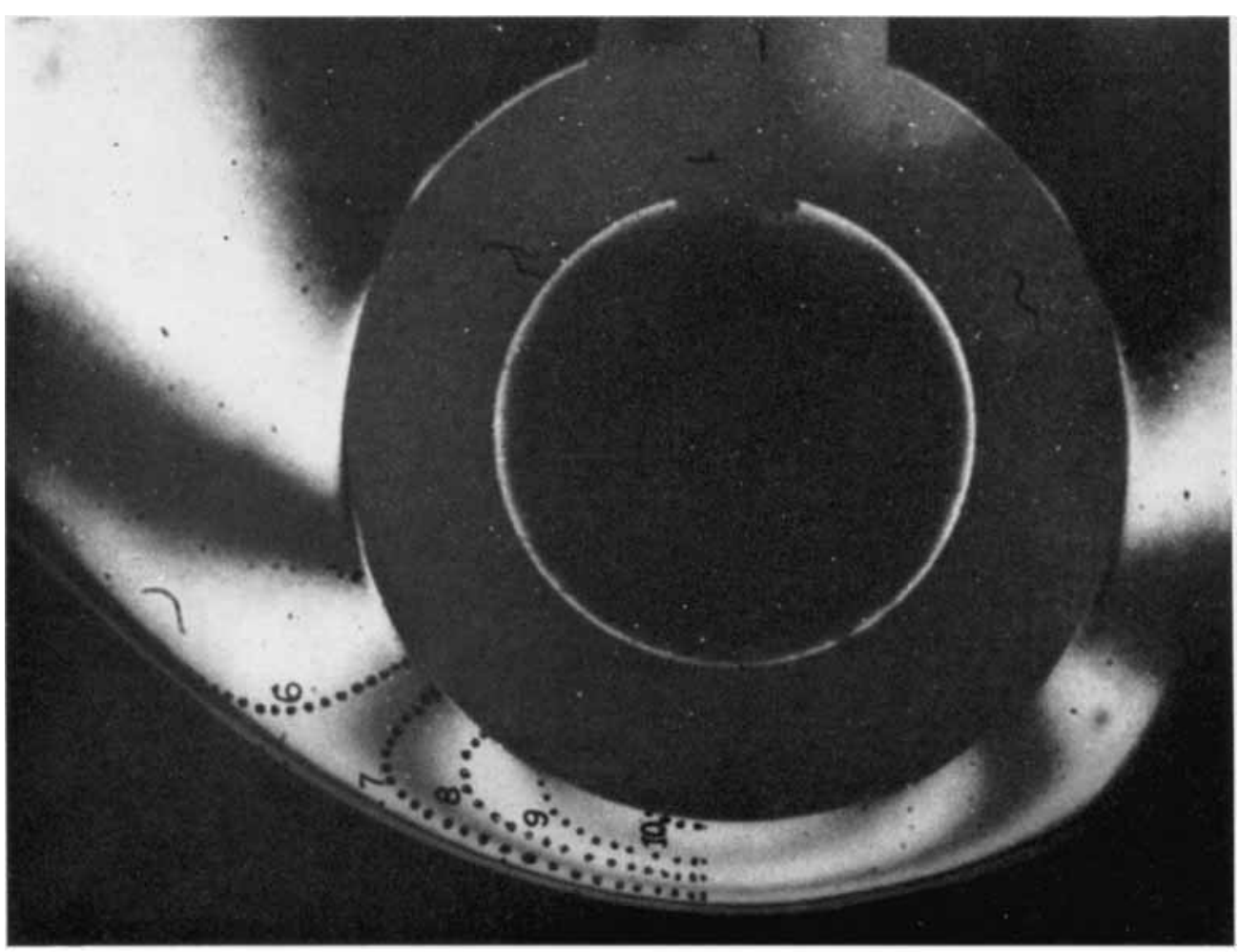

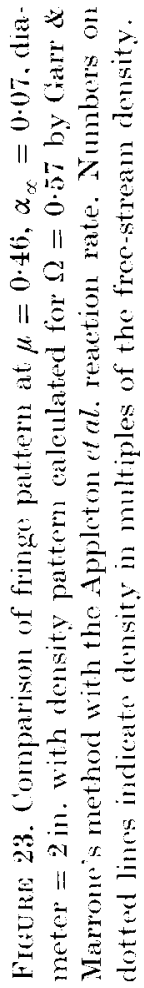
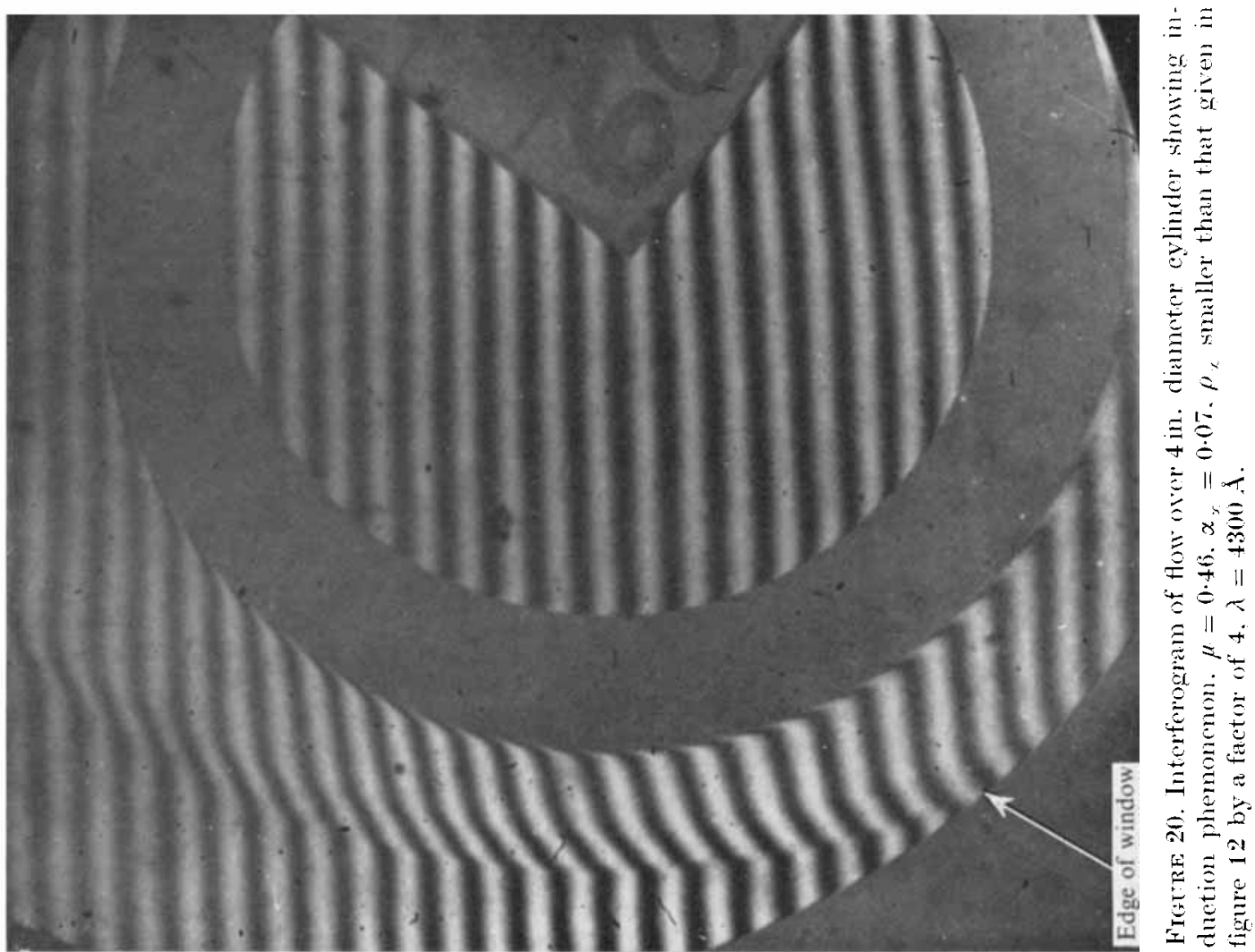